\title{
Article \\ Correlations between the Composition of Liquid Fraction of Full-Scale Digestates and Process Conditions
}

\author{
Afifi Akhiar 1,2,*(D), Felipe Guilayn ${ }^{1,+} \mathbb{D}$, Michel Torrijos ${ }^{1}$, Audrey Battimelli ${ }^{1, *}$, Abd Halim Shamsuddin ${ }^{2} \mathbb{D}$ and \\ Hélène Carrère ${ }^{1}$ (D)
}

1 INRAE, Université de Montpellier, LBE, 102 Avenue des Etangs, 11100 Narbonne, France; felipe.guilayn@suez.com (F.G.); michel.torrijos@inrae.fr (M.T.); helene.carrere@inrae.fr (H.C.)

2 Institute of Sustainable Energy (ISE), Universiti Tenaga Nasional, Jalan IKRAM-UNITEN, Kajang 43000, Selangor, Malaysia; abdhalim@uniten.edu.my

* Correspondence: afifi.akhiar@uniten.edu.my (A.A.); audrey.battimelli@inrae.fr (A.B.); Tel.: +33-468-425-153 (A.B.)

+ Present address: SUEZ, Centre International de Recherche Sur l'Eau et l'Environnement (CIRSEE), 38 rue du Président Wilson, 78230 Le Pecq, France.

Citation: Akhiar, A.; Guilayn, F.; Torrijos, M.; Battimelli, A.;

Shamsuddin, A.H.; Carrère, $\mathrm{H}$.

Correlations between the Composition of Liquid Fraction of Full-Scale Digestates and Process Conditions. Energies 2021, 14, 971. https://doi.org/10.3390/en14040971

Academic Editor: Attilio Converti

Received: 24 November 2020

Accepted: 3 February 2021

Published: 12 February 2021

Publisher's Note: MDPI stays neutral with regard to jurisdictional claims in published maps and institutional affiliations.

Copyright: (c) 2021 by the authors. Licensee MDPI, Basel, Switzerland. This article is an open access article distributed under the terms and conditions of the Creative Commons Attribution (CC BY) license (https:// creativecommons.org/licenses/by/ $4.0 /)$.

\begin{abstract}
Fast development of centralized agricultural biogas plants leads to high amounts of digestate production. The treatment and disposal of liquid fractions after on-site digestate solidliquid separation remains problematic due to their high organic, nutrient and aromatic contents. This work aims to study the variability of the remaining compounds in the digestate liquid fractions in relation to substrate origin, process parameters and solid-liquid separation techniques. Twenty-nine digestates from full-scale codigestion biogas plants and one waste activated sludge (WAS) digestate were collected and characterized. This study highlighted the combined effect of the solid-liquid separation process and the anaerobic digestion feedstock on the characteristics of liquid fractions of digestates. Two major clusters were found: (1) liquid fractions from high efficiency separation process equipment (e.g., centrifuge and others with addition of coagulant, flocculent or polymer) and (2) liquid fractions from low efficiency separation processes (e.g., screw press, vibrating screen and rotary drum), in this latter case, the concentration of chemical oxygen demand (COD) was associated with the proportion of cow manure and energy crops at biogas plant input. Finally, $\mathrm{SUVA}_{254}$, an indicator for aromatic molecule content and the stabilization of organic matter, was associated with the hydraulic retention time (HRT).
\end{abstract}

Keywords: anaerobic digestion; solid waste; organic compound; solid-liquid separation; liquid phase; digestate treatment

\section{Introduction}

The development of biogas plants from agricultural waste in Europe was particularly due to its energy policy to implement Clean Energy Package including Renewable Energy Directive. This policy aims to achieve a 32\% share of renewable energy from total energy consumption by the year 2030 [1-3]. In consequence, this leads to a huge production of biogas plant byproducts, digestate, a renewable resource [4] which requires post-treatment for nutrient recovery to meet the latest European Union regulation proposal on fertilizers [3,5].

The most common current practice of digestate post-treatment is by volume reduction through mechanical solid-liquid separation [6,7]; producing 80-92\% of liquid fraction in terms of mass; common separators on sites are the screw press, centrifuge, vibrating screen or rotary drum [8-10].

Solid fractions of digestates which contain more $\mathrm{P}$ are generally utilized for land application as fertilizer $[6,11,12]$. On the other hand, liquid fractions of digestates still contain high residual of organic compounds with the concentration of total chemical oxygen demand (COD) from 9.2 to $78 \mathrm{~g} / \mathrm{L}$; where 60 to $96 \%$ of COD are in the form of 
suspended particles $(>1.2 \mu \mathrm{m})$ while the remaining are in the form of colloids (1.2 $\mu \mathrm{m}$ to $1 \mathrm{kDa})$ and dissolved matter $(<1 \mathrm{kDa})$, representing $2-27 \%$ and $2-18 \%$, respectively [13]. Owing to its poor aerobic biodegradability characteristics with high humic substance content [13], aerobic post-treatment for liquid fractions of digestates is far from feasible. Besides, the liquid fraction contains high amounts of nutrients such as; total nitrogen (TN) (1.5 to $6.5 \mathrm{~g} / \mathrm{L}), 0.5$ to $3.5 \mathrm{~g} / \mathrm{L}$ for ammoniacal nitrogen $\left(\mathrm{NH}_{4}{ }^{+}\right.$and $\left.\mathrm{NH}_{3}\right), 1.05$ to $5.48 \mathrm{~g} / \mathrm{L}$ for potassium $\left(\mathrm{K}^{+}\right)$and 0 to $2.13 \mathrm{~g} / \mathrm{L}$ for phosphates $\left.\left(\mathrm{PO}_{4}{ }^{3-}\right)\right)[6,12-15]$ which exhibit a fertilizing potential for crops [16].

Currently, new technologies for processing liquid fractions of digestates are still being explored [17]. One of the possibilities is through nutrient recovery such as struvite (STR) precipitation [18] and ammonia stripping (to produce ammonium sulphate (AS)) [6,19-21], combined ozone treatment and ultrafiltration [22], combined system with aerobic granular sludge batch reactors and ultrafiltration [23], or utilizing fly ash as a chemical precipitant [24].

Besides, high nutrient contents mean that the liquid fractions of digestates able to be reused for microalgae cultivation for biomass [25,26] or as biomass for fertilizer [27], recycling nutrients back to digesters, soil application and subsurface injection into soils [6,28].

The appropriate post-treatments for either solid fractions or liquid fractions of digestates are very crucial for any future biogas plant that integrates part of the circular bioeconomy $[17,29,30]$. The aim of the circular economy is to influence material and energy flows in order to maximize environmental benefits whilst avoiding costs (grow-makese-restore) [31]; which is currently one of the main priorities of the European Union as described in detail by Molina-Moreno et al. [32], Muradin et al. [33] and Vilardi et al. [34]. However, one type of full-scale post-treatment could not be applied to all liquid fractions of digestates mainly because the composition in organics, nutrients and aromatic compounds can strongly vary from one liquid fraction of digestate to another.

The primary aim of the research is to understand the variability of the remaining compounds in the liquid fractions of digestates specifically produced at full-scale codigestion plants in relation with substrates origin, operating conditions of the digester and types of solid-liquid separation. For the first time, a substantial number of liquid fractions (29) were sampled from full-scale anaerobic codigestion plants treating agricultural wastes and then deeply characterized. A single liquid fraction of digestate from a common anaerobic digestion plant treating waste activated sludge (WAS) was also collected as a benchmark for agricultural codigestion plants.

\section{Materials and Methods}

\subsection{Digestate Collection and Storage}

Digestates (raw digestate, solid and liquid fractions of digestates after separation) were taken from 30 full-scale anaerobic digestion plants. Eleven samples were already described in a previous paper [13]; see the plant reference marked with an asterisk in Table 1. Two liters of each raw digestate and solid fraction of digestate, respectively, and $4 \mathrm{~L}$ of liquid fraction of digestate were collected from each plant for this study. In this investigation, raw digestate and solid fraction of digestate were collected for analyses of total solids (TS) and volatile solids (VS) concentrations in order to gain information on solid-liquid separation efficiency performed on-site. All samples were stored in a cold room at $4{ }^{\circ} \mathrm{C}$ for later use.

\subsection{Categorization of Substrates}

The different substrates used to feed the different digesters were distributed into 7 main categories: sewage sludge (SS), manure (Mnr), energy crops (EnCr), crop residues (CrR), cereal residues (Cer), fats, oil and grease (FOG) and agro-food waste (AFW) (Table 1). The different types of substrates used to feed the digesters based on the selected categories are described in Table A1. 


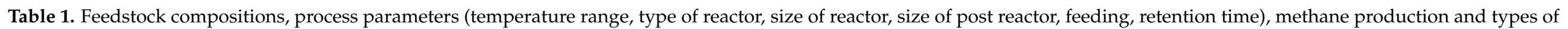
solid-liquid separation.

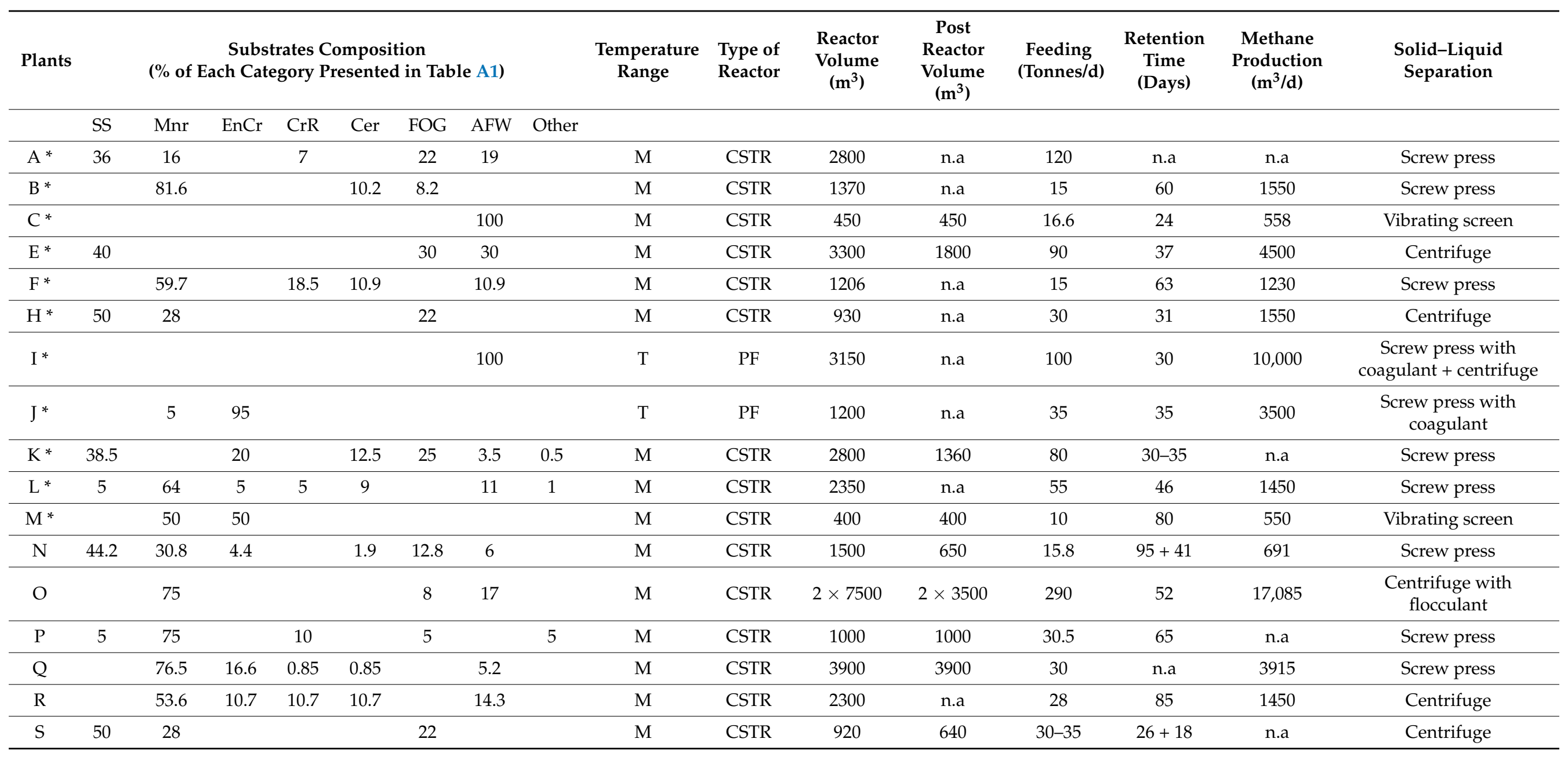


Table 1. Cont.

\begin{tabular}{|c|c|c|c|c|c|c|c|c|c|c|c|c|c|c|}
\hline $\begin{array}{c}\text { Plants } \\
\mathrm{T}\end{array}$ & \multicolumn{6}{|c|}{$\begin{array}{c}\text { Substrates Composition } \\
\text { (\% of Each Category Presented in Table A1) }\end{array}$} & $\begin{array}{c}\begin{array}{c}\text { Temperature } \\
\text { Range }\end{array} \\
\mathrm{M}\end{array}$ & $\begin{array}{l}\text { Type of } \\
\text { Reactor } \\
\text { CSTR }\end{array}$ & $\begin{array}{c}\begin{array}{c}\text { Reactor } \\
\text { Volume } \\
\left(\mathrm{m}^{3}\right)\end{array} \\
2600\end{array}$ & $\begin{array}{c}\begin{array}{c}\text { Post } \\
\text { Reactor }\end{array} \\
\begin{array}{c}\text { Volume } \\
\left(\mathrm{m}^{3}\right)\end{array} \\
\text { n.a }\end{array}$ & $\begin{array}{c}\begin{array}{c}\text { Feeding } \\
\text { (Tonnes/d) }\end{array} \\
34.2\end{array}$ & $\begin{array}{c}\begin{array}{c}\text { Retention } \\
\text { Time } \\
\text { (Days) }\end{array} \\
50\end{array}$ & $\begin{array}{c}\begin{array}{c}\text { Methane } \\
\text { Production } \\
\left(\mathbf{m}^{3} / \mathrm{d}\right)\end{array} \\
2381\end{array}$ & $\begin{array}{c}\begin{array}{c}\text { Solid-Liquid } \\
\text { Separation }\end{array} \\
\begin{array}{c}\text { Rotary drum. Solid } \\
\text { fraction was dried }\end{array}\end{array}$ \\
\hline $\mathrm{U}$ & & 55.5 & 42.1 & 2.4 & & & $40-45$ & CSTR & $2 \times 718.5$ & 682 & $2 \times 10.6$ & $(2 \times 68)+32.5$ & n.a & Screw press \\
\hline V & & 36.2 & 56.9 & 6.9 & & & $\mathrm{~T}$ & CSTR & $2 \times 1500$ & 3000 & 29 & 37 & n.a & Screw press \\
\hline$x$ & & & 100 & & & & $40-41$ & CSTR & 2400 & n.a & $27-30$ & $70-80$ & n.a & Screw press \\
\hline $\mathrm{Y}$ & & & & 4.7 & 25.7 & 69.6 & M & CSTR & 3400 & 1600 & $\begin{array}{l}57+35 \text { re- } \\
\text { circulation }\end{array}$ & 37 & 12,400 & Screw press \\
\hline $\mathrm{Z}$ & & 82 & 13 & 5 & & & M & CSTR & 1200 & 1200 & 31.3 & 66 & 1418 & Screw press \\
\hline $\mathrm{I} 2$ & & & & & & 100 & $\mathrm{~T}$ & PF & 3150 & n.a & $100-150$ & $20-30$ & $5500-8500$ & $\begin{array}{l}\text { Screw press with } \\
\text { flocculant }+ \text { centrifuge }\end{array}$ \\
\hline $\mathrm{AA}$ & & 60.1 & 17.9 & 6.2 & 15.8 & & M & CSTR & 1300 & n.a & 65.9 & 45 & 2300 & Screw press \\
\hline $\mathrm{AB}$ & & 33 & 20 & & 20 & 27 & M & CSTR & 2900 & n.a & 50 & 57 & 3090 & Screw press \\
\hline $\mathrm{AC}$ & & 100 & & & & & $\mathrm{M}$ & CSTR & 500 & n.a & $7-8$ & 40 & n.a & Centrifuge \\
\hline $\mathrm{AD}$ & 48 & & & & 4 & 48 & $\mathrm{M}$ & CSTR & 1500 & 3000 & 75 & $20+40$ & 2790 & $\begin{array}{c}\text { Filter press (150 plates) + } \\
\text { inorganic coagulant + } \\
\text { polymer. Solid fraction } \\
\text { was later dried }\end{array}$ \\
\hline G & 100 & & & & & & M & CSTR & 10,000 & n.a & 19.1 & 20 & 5583 & $\begin{array}{c}\text { Centrifuge with addition } \\
\text { of polymer }\end{array}$ \\
\hline
\end{tabular}

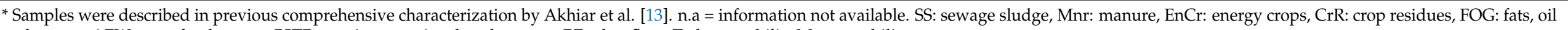
and grease, AFW: agro-food waste. CSTR: continuous stirred-tank reactor, PF: plug flow. T: thermophilic, M: mesophilic. 


\subsection{Operating Conditions of the Anaerobic Plants}

Details on substrate composition, operating parameters and solid-liquid separation of the different plants are presented in Table 1. In this study, digestates (raw, solid and liquid fractions) from an ordinary anaerobic digestion plant fed with only Waste Activated Sludge (WAS) (Plant G) were also collected in order to compare with samples from codigestion plants.

\subsection{Filtration and Size Fractionation of Liquid Fractions of Digestates}

Dilution with Milli- $Q^{\circledR}$ water was initially performed on each respective liquid fraction of digestate to ease filtration. Dilution factor from 0 to $1 / 20$ was considered in order to have a final COD concentration ranged between 1 to $5 \mathrm{~g} / \mathrm{L}$. Filtration at size $1.2 \mu \mathrm{m}$ and $1 \mathrm{kDa}$ performed later on each respective liquid fraction of digestate enables us to have four fractionation sizes representing: raw liquid (without any filtration), suspended particles (size $>1.2 \mu \mathrm{m}$ ), colloids (size $1.2 \mu \mathrm{m}^{-1} \mathrm{kDa}$ ) and dissolved matter (size $<1 \mathrm{kDa}$ ) [35].

\subsection{Analytical Methods (Chemical, Physical and Biological)}

The following analytical methods were similar to procedures performed (where detailed description can be found) in previous work by Akhiar et al. [13].

For chemical methods, a WTW series inoLab $\mathrm{pH} 720$ probe was used for $\mathrm{pH}$ measurement. Calibration with $\mathrm{pH} 4$ and $\mathrm{pH} 7$ buffer solutions was mandatory prior to use. For alkalinity, $0.1 \mathrm{~N}$ hydrochloric acid was used for titration to reach $\mathrm{pH} 4.3$ as described elsewhere [36]. Total solids (TS), volatile solids (VS) and mineral solids (MS) analyses were performed according to standard methods described elsewhere [36]. Commercial Aqualytic $^{\text {TM }} 420721$ COD Vario Tube Test MR 0-1500 mg/L (Aqualytic, Dortmund, Germany) was used to measure Chemical Oxygen Demand (COD). Buchi AutoKjeldahl Unit K-370 (Büchi AG, Flawil, Switzerland) was used for Ammonium $\left(\mathrm{NH}_{4}{ }^{+}\right)$and Total Kjeldahl Nitrogen (TKN) measurements. For TKN measurement only, premineralization with BUCHI Digest Automat K-438 (Büchi AG, Flawil, Switzerland) was required. Shimadzu TOC-VCSN Total Organic Carbon Analyzer (Shimadzu Corporation, Kyoto, Japan) equipped with Shimadzu ASI-V auto sampler- was utilized for Total Organic Carbon (TOC) and Inorganic Carbon (IC) measurement [37]. Ion chromatograph, ICS 3000 (Dionex, Sunnyvale, CA, USA) was used to measure cations and anions [38].

UV-2501PC UV-vis spectrophotometer (Shimadzu Corporation, Kyoto, Japan) was used to measure absorbance spectra [37]. Specific Ultraviolet Absorbance at $254 \mathrm{~nm}$ $\left(\mathrm{SUVA}_{254}\right)$ was calculated by dividing specific UV absorbance at wavelength $254 \mathrm{~nm}$ with dissolved total organic carbon $\left(\mathrm{A}_{254} / \mathrm{TOC}\right)$. $\mathrm{SUVA}_{254}$ indicates the content of aromatic carbon in dissolved organic matter and humification degree as well as linked to biological degradability [39]. Perkin Elmer LS55 fluorescence spectrometer (PerkinElmer, Waltham, MA, USA) was used for 3D fluorescence spectroscopy analysis. Fluorescence spatialization integration for spectra interpretation and quantification was according to: (1) protein-like (Tyrosine, Tyrptophane, microbial products); (2) fulvic acid-like; (3) glycolated protein-like; (4) melanoidin-like; lignocellulose-like; (5) Humic acid-like [40].

For physical methods, Beckman Coulter LS200 granulometer (Beckman Coulter, Pasadena, CA, USA) was utilized for the measurement of particle size distribution in the size range between 0 to $2000 \mu \mathrm{m}$ [41]. HACH portable turbidimeter model 2100P (Hach, Loveland, CO, USA) precalibrated with formazin was used to measure turbidity. WTW multi 3410 digital multi parameter meter TretraCon ${ }^{\circledR} 925$ probe (Xylem, Rye Brook, NY, USA) with was used for conductivity measurement at a fixed reference temperature of $25^{\circ} \mathrm{C}$. For biological method, WTW Oxitop ${ }^{\circledR}$ control system (Xylem, Rye Brook, NY, USA) was used for determination of Biochemical Oxygen Demand after 5 days $\left(\mathrm{BOD}_{5}\right)$ and 21 days $\left(\mathrm{BOD}_{21}\right)[42,43]$.

Capillary Suction Time (CST) which measures filterability and conditionability of a given liquid sample containing suspended and colloidal particles was conducted using Type 304B CST timer (Tritonel, Strmec, Croatia) equipped with funnel (18 mm diameter) 
and filter papers (basis weight of $440 \mathrm{~g} / \mathrm{m}^{2}$, size $7 \times 9 \mathrm{~cm}$, thickness of $0.92 \mathrm{~mm}$, tensile strength of $4525 \mathrm{~m} / \mathrm{d} \mathrm{g} / 15 \mathrm{~mm}$, porosity of $9 \mathrm{~s} / 100 \mathrm{~mL} / \mathrm{sqin}$ ) purchased from Triton Electronics Ltd. (Dunmow, UK). Each respective liquid fraction of digestate was prediluted to same TS concentration of $10 \mathrm{~g}$ TS/ $\mathrm{kg}$ and only $2 \mathrm{~mL}$ of diluted sample were used for each analysis.

The analytical results from chemical, physical and biological analyses of 18 samples combined with another 11 samples (samples A, B, C, E, F, H, I, J, K, L, M) from Akhiar et al. [13] and 1 sample from WAS (sample G) are displayed in Appendix A (Tables A2-A4). All the analytical results were used for statistical analysis in this study.

\subsection{Determination of Solid-liquid Separation Efficiency}

The separation efficiency indicates the removal efficiency $(R)$ of a particular compound from a slurry to the solid fraction. The calculation for separation efficiency or removal efficiency $(\mathrm{R})$ by solid-liquid separation techniques was made using Equation (1) below [44].

$$
R=1-\frac{[T S] \text { liq }}{[T S] \text { raw }}
$$

where $[T S]_{l i q}=$ total solids concentration in liquid fraction of digestate and $[T S]_{\text {raw }}=$ total solids concentration in raw digestate.

\subsection{Statistical Analysis}

The classification of the parameters analyzed on liquid fractions of digestates from 29 codigestion plants and 1 WAS plant was performed via Principal component analysis (PCA), hierarchical cluster analysis (HCA) and correlation matrix using $\mathrm{R}$ version 3.3.2 (31 December 2016) [45]. PCA was carried out in center-scaled variables using function 'FactoMineR' package version 1.35 [46] with PCA plots package 'factoextra' version 1.0.4. For HCA, 'stats' package version 3.3.2 ('hclust' function) was applied to center-scaled variables and Euclidean distances. The clustering algorithm was referring to Ward [47] and the resulting dendrogram was plotted using function 'dendextend' package version 1.4.0. Meanwhile, correlation matrices were constructed using 'rcorr' function with the Pearson's correlation method ('rcorr' is a function of the 'Hmisc' package (version 4.0.2)).

\section{Results and Discussion}

All results from chemical, physical and biological analyses performed on liquid fractions of digestates are presented in Tables A2-A4. The feedstocks used, the operating parameters (types of reactor, temperature, loading rate, hydraulic retention time (HRT), methane production) and types of solid-liquid separation equipment used are presented in Table 1. The chemical, physical and biological characteristics of the liquid fractions of digestates analyzed (based on the following fractions: raw liquid, suspended particles, colloids and dissolved matter) were included in the PCA, HCA and correlation matrix.

\subsection{Correlation between Parameters}

Only significant correlations between parameters are shown in Table 2 ( $p$-value $<0.01)$. In relation to feedstock composition, TKN in colloids was clearly observed to correlate with sewage sludge in the feeding. Indeed, sewage sludge contains very high TN based on dry matter basis due to low total solids content of the sludge after efficient centrifugation. In a study by Oliveira et al. [48], high correlations between nitrogen content in the digestate and both sludge composition or conditioning parameters were reported. Similar to this study, it was observed that as the sewage sludge proportion increased at the feed, higher colloidal TKN in liquid fractions of the digestates was observed. In comparison, none of the other feedstock categories have shown high correlation with the characteristics of liquid fractions of digestates. This remark may be supported by the high uncertainty of the quantities reported from the full-scale plants, the lack of detailed information such as VS quantities in the feeding (instead of total mass) but also by the selection of categories that might not 
be specific enough (i.e., there is a high variation of quality within feedstocks of the same category). Moreover, several studies reported high variabilities of digestate from the same plant over time but also that anaerobic digestion acts as a buffer for feedstock variation, producing digestates with less quality variability than inputs $[49,50]$. Both effects would tend to reduce correlation observation based on single samples from different plants.

Table 2. Summary of correlations ( $p$-value $<0.01$ ).

\begin{tabular}{|c|c|c|c|c|c|}
\hline \multirow{2}{*}{ Parameters } & \multirow{2}{*}{ Unit } & \multicolumn{2}{|c|}{ Strong (Anti-)Correlation } & \multicolumn{2}{|c|}{ Moderate (Anti-)Correlation } \\
\hline & & $|\mathrm{r}|>0.7$ & $\mathbf{r}$ & $0.5<|\mathrm{r}|<0.7$ & $\mathbf{r}$ \\
\hline Sewage Sludge a & $w / w$ & TKN colloids & 0.74 & $\begin{array}{c}\text { Alkalinity } \\
\text { IC } \\
\text { TOC dissolved } \\
\text { TKN total } \\
\mathrm{NH}_{4}^{+}\end{array}$ & $\begin{array}{c}0.5 \\
0.51 \\
0.54 \\
0.52 \\
0.52\end{array}$ \\
\hline $\mathrm{EnCr}{ }^{\mathrm{a}}$ & $w / w$ & & & $\begin{array}{l}\text { VS/TS liquid } \\
\text { MS/TS liquid } \\
\text { SUVA }_{254}\end{array}$ & $\begin{array}{c}0.51 \\
-0.51 \\
0.67\end{array}$ \\
\hline Cer $^{a}$ & $w / w$ & & & CST & 0.67 \\
\hline $\mathrm{AFW}^{\mathrm{a}}$ & $w / w$ & $\begin{array}{l}\text { MS/TS raw } \\
\text { VS/TS raw }\end{array}$ & $\begin{array}{c}0.70 \\
-0.70\end{array}$ & $\begin{array}{l}\text { VS/TS liquid } \\
\text { MS/TS liquid }\end{array}$ & $\begin{array}{c}-0.52 \\
0.52 \\
\end{array}$ \\
\hline Load & $\mathrm{t} /$ day $/ \mathrm{m}^{3}$ reactor & & & AFW & 0.54 \\
\hline HRT & Days & $\mathrm{SUVA}_{254}$ & 0.72 & $\begin{array}{c}\text { CST } \\
\text { TOC dissolved } \\
\text { Turbidity } \\
\text { C/N }\end{array}$ & $\begin{array}{c}0.64 \\
-0.5 \\
0.5 \\
0.55\end{array}$ \\
\hline Methane production & $\mathrm{m}^{3} \mathrm{CH}_{4} /$ ton fed & & & TKN colloids & 0.67 \\
\hline VS/TS raw digestate & $w / w$ & & & $\begin{array}{c}\text { VS/TS liquid } \\
\text { MS/TS liquid } \\
\text { COD total } \\
\text { COD suspended }\end{array}$ & $\begin{array}{c}0.63 \\
-0.63 \\
0.57 \\
0.51\end{array}$ \\
\hline MS/TS raw digestate & $w / w$ & VS/TS raw & -1 & $\begin{array}{c}\text { VS/TS liquid } \\
\text { MS/TS liquid } \\
\text { COD total } \\
\text { COD suspended }\end{array}$ & $\begin{array}{c}-0.63 \\
0.63 \\
-0.57 \\
-0.51\end{array}$ \\
\hline VS/TS solid fraction & $w / w$ & $\begin{array}{c}\text { MS/TS solids } \\
\text { Separ. Efficiency }\end{array}$ & $\begin{array}{l}-0.99 \\
-0.72\end{array}$ & $\begin{array}{c}\text { MS/TS raw } \\
\text { VS/TS raw } \\
\text { COD suspended } \\
\text { COD dissolved } \\
\text { Turbidity } \\
\mathrm{C} / \mathrm{N} \\
\mathrm{Na}^{+} \\
\end{array}$ & $\begin{array}{c}-0.53 \\
0.53 \\
0.51 \\
-0.51 \\
0.64 \\
0.5 \\
-0.60 \\
\end{array}$ \\
\hline MS/TS solid fraction & $w / w$ & Separ. Efficiency & 0.74 & $\begin{array}{c}\text { MS/TS raw } \\
\text { VS/TS raw } \\
\text { COD dissolved } \\
\text { Turbidity } \\
\text { Turbidity } \\
\mathrm{Na}^{+}\end{array}$ & $\begin{array}{c}0.5 \\
-0.5 \\
0.52 \\
-0.63 \\
-0.63 \\
0.60\end{array}$ \\
\hline VS/TS liquid fraction & $w / w$ & $\begin{array}{l}\text { MS/TS liquid } \\
\text { COD total } \\
\text { COD suspended }\end{array}$ & $\begin{array}{c}- \\
10.79 \\
0.74\end{array}$ & $\begin{array}{c}\mathrm{CST} \\
\text { Turbidity } \\
\mathrm{C} / \mathrm{N} \\
\mathrm{N} \text { organic dissolved } \\
\mathrm{Na}^{+} \\
\mathrm{Cl}^{-} \\
\text {Conductivity } \\
\text { SUVA }_{254}\end{array}$ & $\begin{array}{c}0.57 \\
0.61 \\
0.57 \\
-0.51 \\
-0.63 \\
-0.56 \\
-0.5 \\
0.56\end{array}$ \\
\hline
\end{tabular}


Table 2. Cont.

\begin{tabular}{|c|c|c|c|c|c|}
\hline \multirow{2}{*}{ Parameters } & \multirow{2}{*}{ Unit } & \multicolumn{2}{|c|}{ Strong (Anti-)Correlation } & \multicolumn{2}{|c|}{ Moderate (Anti-)Correlation } \\
\hline & & $|r|>0.7$ & $\mathbf{r}$ & $0.5<|\mathrm{r}|<0.7$ & $\mathbf{r}$ \\
\hline MS/TS liquid fraction & $w / w$ & $\begin{array}{c}\text { COD total } \\
\text { COD suspended }\end{array}$ & $\begin{array}{l}-0.79 \\
-0.74\end{array}$ & $\begin{array}{c}\mathrm{CST} \\
\text { Turbidity } \\
\mathrm{C} / \mathrm{N} \\
\mathrm{N} \text { organic dissolved } \\
\mathrm{Na}^{+} \\
\mathrm{Cl}^{-} \\
\text {Conductivity } \\
\text { SUVA }_{254}\end{array}$ & $\begin{array}{c}-0.57 \\
-0.61 \\
-0.57 \\
0.51 \\
0.63 \\
0.56 \\
0.5 \\
-0.56\end{array}$ \\
\hline Separation efficiency & $w / w$ & $\begin{array}{l}\text { COD dissolved } \\
\text { Turbidity } \\
\text { TKN dissolved } \\
\text { Conductivity } \\
\mathrm{NH}_{4}^{+}\end{array}$ & $\begin{array}{c}0.77 \\
-0.70 \\
0.70 \\
0.72 \\
0.7\end{array}$ & $\begin{array}{c}\text { CST } \\
\text { Alkalinity } \\
\text { IC } \\
\text { TOC dissolved } \\
\text { C/N } \\
\text { TKN total } \\
\text { TKN colloids } \\
\text { N organic dissolved }\end{array}$ & $\begin{array}{c}-0.60 \\
0.64 \\
0.68 \\
0.61 \\
-0.55 \\
0.69 \\
0.65 \\
0.57\end{array}$ \\
\hline CST & Seconds & & & $\begin{array}{l}\text { COD suspended } \\
\text { Turbidity } \\
\text { Conductivity } \\
\text { SUVA }_{254}\end{array}$ & $\begin{array}{c}0.56 \\
0.60 \\
-0.56 \\
0.68\end{array}$ \\
\hline $\mathrm{pH}$ & - & & & $\begin{array}{l}\text { COD suspended } \\
\mathrm{C} / \mathrm{N} \\
\mathrm{Na}^{+} \\
\mathrm{Cl}^{-} \\
\text {Glycolated-like } \\
\text { Humic acid-like }\end{array}$ & $\begin{array}{c}-0.53 \\
-0.51 \\
0.57 \\
0.63 \\
0.53 \\
0.54\end{array}$ \\
\hline Alkalinity & $\mathrm{gCaCO}_{3} / \mathrm{gTS}$ & $\begin{array}{c}\text { IC } \\
\text { TOC dissolved } \\
\text { C/N } \\
\text { TKN total } \\
\text { TKN suspended } \\
\text { TKN colloids } \\
\text { TKN dissolved } \\
\text { N organic } \\
\text { dissolved } \\
\mathrm{NH}_{4}^{+} \\
\text {Conductivity }\end{array}$ & $\begin{array}{c}0.97 \\
0.71 \\
-0.71 \\
0.97 \\
0.81 \\
0.71 \\
0.94 \\
0.8 \\
0.93 \\
0.91\end{array}$ & & \\
\hline IC & $\mathrm{gC} / \mathrm{gTS}$ & $\begin{array}{c}\text { TOC dissolved } \\
\text { C/N } \\
\text { TKN total } \\
\text { TKN suspended } \\
\text { TKN colloids } \\
\text { TKN dissolved } \\
\text { N organic } \\
\text { dissolved } \\
\mathrm{NH}_{4}^{+} \\
\text {Conductivity }\end{array}$ & $\begin{array}{c}0.79 \\
-0.75 \\
0.97 \\
0.74 \\
0.73 \\
0.95 \\
0.79 \\
0.94 \\
0.94\end{array}$ & $\begin{array}{l}\text { COD dissolved } \\
\text { Turbidity } \\
\text { SUVA }_{254}\end{array}$ & $\begin{array}{l}0.55 \\
-0.5 \\
0.51\end{array}$ \\
\hline Conductivity & $(\mathrm{mS} / \mathrm{cm}) /(\mathrm{gTS} / \mathrm{kg})$ & & & $\mathrm{SUVA}_{254}$ & -0.5 \\
\hline
\end{tabular}


Table 2. Cont.

\begin{tabular}{|c|c|c|c|c|c|}
\hline \multirow{2}{*}{ Parameters } & \multirow{2}{*}{ Unit } & \multicolumn{2}{|c|}{ Strong (Anti-)Correlation } & \multicolumn{2}{|c|}{ Moderate (Anti-)Correlation } \\
\hline & & $|\mathrm{r}|>0.7$ & $\mathbf{r}$ & $0.5<|\mathrm{r}|<0.7$ & $\mathbf{r}$ \\
\hline TOC dissolved & $\mathrm{gC} / \mathrm{gTS}$ & & & $\begin{array}{c}\text { COD dissolved } \\
\mathrm{C} / \mathrm{N} \\
\text { TKN total } \\
\text { TKN suspended } \\
\text { TKN colloids } \\
\text { TKN dissolved } \\
\mathrm{N} \text { organic dissolved } \\
\mathrm{NH}_{4}{ }^{+} \\
\mathrm{PO}_{4}{ }^{3-} \\
\text { Conductivity } \\
\text { SUVA }_{254}\end{array}$ & $\begin{array}{c}0.52 \\
-0.61 \\
0.67 \\
0.62 \\
0.66 \\
0.62 \\
0.52 \\
0.61 \\
0.61 \\
0.66 \\
-0.65\end{array}$ \\
\hline COD total & $\mathrm{gO}_{2} / \mathrm{gTS}$ & COD suspended & 0.93 & $\begin{array}{c}\text { Turbidity } \\
\mathrm{Na}^{+} \\
\mathrm{Cl}^{-} \\
\mathrm{BOD}_{21} \\
\end{array}$ & $\begin{array}{c}0.57 \\
-0.64 \\
-0.67 \\
0.60 \\
\end{array}$ \\
\hline COD suspended & $\mathrm{gO}_{2} / \mathrm{gTS}$ & $\begin{array}{l}\text { Turbidity } \\
\mathrm{Cl}^{-}\end{array}$ & $\begin{array}{c}0.76 \\
-0.73\end{array}$ & $\begin{array}{c}\mathrm{C} / \mathrm{N} \\
\mathrm{Na}^{+} \\
\text {Conductivity } \\
\mathrm{BOD}_{21}\end{array}$ & $\begin{array}{c}0.52 \\
-0.66 \\
-0.57 \\
0.5\end{array}$ \\
\hline COD colloids & $\mathrm{gO}_{2} / \mathrm{gTS}$ & & & COD dissolved & 0.66 \\
\hline COD dissolved & $\mathrm{gO}_{2} / \mathrm{gTS}$ & & & $\begin{array}{c}\text { Turbidity } \\
\mathrm{C} / \mathrm{N} \\
\mathrm{TKN} \text { total } \\
\text { TKN dissolved } \\
\mathrm{N} \text { organic dissolved } \\
\mathrm{NH}_{4}^{+} \\
\mathrm{K}^{+} \\
\text {Conductivity }\end{array}$ & $\begin{array}{c}-0.57 \\
-0.53 \\
0.52 \\
0.53 \\
0.51 \\
0.5 \\
0.51 \\
0.57\end{array}$ \\
\hline Turbidity & NTU/(gTS/kg) & & & $\begin{array}{c}\mathrm{C} / \mathrm{N} \\
\mathrm{TKN} \text { total } \\
\text { TKN dissolved } \\
\mathrm{N} \text { organic dissolved } \\
\mathrm{NH}_{4}^{+} \\
\mathrm{Na}^{+} \\
\mathrm{Cl}^{-} \\
\text {Conductivity }\end{array}$ & $\begin{array}{l}0.57 \\
-0.5 \\
-0.55 \\
-0.55 \\
-0.53 \\
-0.62 \\
-0.69 \\
-0.64\end{array}$ \\
\hline $\mathrm{C} / \mathrm{N}$ & - & $\begin{array}{c}\text { TKN total } \\
\text { TKN dissolved } \\
\mathrm{NH}_{4}{ }^{+} \text {Conductivity }\end{array}$ & $\begin{array}{l}-0.74 \\
-0.73 \\
-0.71 \\
-0.78\end{array}$ & $\begin{array}{c}\text { TKN suspended } \\
\text { TC/TN dissolved } \\
\text { N organic dissolved } \\
\mathrm{Na}^{+} \\
\mathrm{SUVA}_{254}\end{array}$ & $\begin{array}{c}-0.58 \\
0.54 \\
-0.67 \\
-0.55 \\
0.54\end{array}$ \\
\hline TKN suspended & $\mathrm{gN} / \mathrm{gTS}$ & & & $\begin{array}{c}\text { TKN colloids } \\
\text { TKN dissolved } \\
\text { N organic dissolved } \\
\mathrm{NH}_{4}^{+} \\
\text {Conductivity }\end{array}$ & $\begin{array}{l}0.52 \\
0.62 \\
0.62 \\
0.59 \\
0.62\end{array}$ \\
\hline TKN total & gN/gTS & $\begin{array}{c}\text { TKN suspended } \\
\text { TKN colloids } \\
\text { TKN dissolved } \\
\text { N organic nitrogen } \\
\mathrm{NH}_{4}^{+} \\
\text {Conductivity }\end{array}$ & $\begin{array}{l}0.73 \\
0.76 \\
0.98 \\
0.81 \\
0.97 \\
0.97\end{array}$ & & \\
\hline
\end{tabular}


Table 2. Cont

\begin{tabular}{|c|c|c|c|c|c|}
\hline \multirow{2}{*}{ Parameters } & \multirow{2}{*}{ Unit } & \multicolumn{2}{|c|}{ Strong (Anti-)Correlation } & \multicolumn{2}{|c|}{ Moderate (Anti-)Correlation } \\
\hline & & $|\mathrm{r}|>0.7$ & $\mathbf{r}$ & $0.5<|\mathrm{r}|<0.7$ & $\mathbf{r}$ \\
\hline TKN colloids & $\mathrm{gN} / \mathrm{gTS}$ & $\begin{array}{c}\mathrm{NH}_{4}^{+} \\
\text {Conductivity }\end{array}$ & $\begin{array}{l}0.74 \\
0.71\end{array}$ & TKN dissolved & 0.69 \\
\hline TKN dissolved & $\mathrm{gN} / \mathrm{gTS}$ & $\begin{array}{c}\mathrm{N} \text { organic } \\
\text { dissolved } \\
\mathrm{NH}_{4}^{+} \\
\text {Conductivity }\end{array}$ & $\begin{array}{l}0.83 \\
0.99 \\
0.97\end{array}$ & $\begin{array}{c}\mathrm{TC} / \mathrm{TN} \text { dissolved } \\
\mathrm{Cl}^{-}\end{array}$ & $\begin{array}{c}-0.51 \\
0.5\end{array}$ \\
\hline $\mathrm{TC} / \mathrm{TN}$ dissolved & - & & & $\begin{array}{c}\mathrm{NH}_{4}^{+} \\
\text {Protein-like } \\
\text { Fulvic acid-like }\end{array}$ & $\begin{array}{c}-0.51 \\
0.56 \\
-0.58\end{array}$ \\
\hline $\mathrm{N}$ organic dissolved & $\mathrm{gN} / \mathrm{gTS}$ & $\begin{array}{c}\mathrm{NH}_{4}^{+} \\
\text {Conductivity }\end{array}$ & $\begin{array}{l}0.74 \\
0.84\end{array}$ & $\begin{array}{c}\mathrm{Na}^{+} \\
\mathrm{K}^{+} \\
\mathrm{Cl}^{-}\end{array}$ & $\begin{array}{l}0.65 \\
0.51 \\
0.55\end{array}$ \\
\hline $\mathrm{NH}_{4}^{+}$ & $\mathrm{gN} / \mathrm{gTS}$ & Conductivity & 0.96 & & \\
\hline $\mathrm{Na}^{+}$ & $\mathrm{gNa} / \mathrm{gTS}$ & & & $\begin{array}{c}\mathrm{Cl}^{-} \\
\mathrm{PO}_{4}^{3-} \\
\text { Conductivity } \\
\text { Humic acid-like }\end{array}$ & $\begin{array}{l}0.67 \\
0.55 \\
0.55 \\
0.54\end{array}$ \\
\hline $\mathrm{Cl}^{-}$ & $\mathrm{gCl} / \mathrm{gTS}$ & & & $\begin{array}{l}\text { Conductivity } \\
\text { Protein-like } \\
\text { Glycolated-like }\end{array}$ & $\begin{array}{c}0.58 \\
-0.61 \\
0.59\end{array}$ \\
\hline Mean Size & $\mu \mathrm{m}$ & Median size & 0.89 & & \\
\hline $\mathrm{BOD}_{5}$ & $\mathrm{gO}_{2} / \mathrm{TS}$ & $\mathrm{BOD}_{21}$ & 0.85 & & \\
\hline Protein-like & - & $\begin{array}{l}\text { Fulvic acid-like } \\
\text { Glycolated-like } \\
\text { Melanoidin-like }\end{array}$ & $\begin{array}{l}-0.88 \\
-0.84 \\
-0.72\end{array}$ & & \\
\hline Glycolated-like & - & Melanoidin-like & 0.71 & Humic acid-like & 0.5 \\
\hline Fulvic acid-like & - & & & Glycolated-like & 0.5 \\
\hline
\end{tabular}

a. AD feedstock proportion.

An anticorrelation was observed between residues of AFW and VS/TS in the raw digestates. This observation may possibly be justified by the characteristics of these feedstocks which are highly biodegradable. This leads to a lower organic matter content (VS/TS) after anaerobic digestion.

The correlation matrix highlights several high correlations between anaerobic process parameters. Specifically, HRT was observed to have a positive correlation with $\mathrm{SUVA}_{254}$. This signifies that higher HRT used will result to a higher humification ratio. This statement will be further discussed in detail in Section 3.4.

From the observation of strong (anti-) correlations between characterization parameters $(|\mathrm{r}|>0.7)$, VS/TS on solid fraction was anticorrelated with separation efficiency which can be linked to the fact that low performance separators are applied mostly to digestates with a high content of fibers that present a higher VS/TS ratio.

For liquid fractions of digestates, VS/TS was correlated to total and suspended COD in liquid fractions of digestates. Total COD was correlated with suspended COD which confirmed the finding by Akhiar et al. [13] that 60-96\% of COD in liquid fractions of digestates are mainly in the form of suspended particles $(>1.2 \mu \mathrm{m})$. Meanwhile, suspended $\mathrm{COD}$ was correlated with turbidity and anticorrelated with $\mathrm{Cl}$. Separation efficiency was also observed to be correlated with dissolved COD, dissolved TKN, conductivity, $\mathrm{NH}_{4}{ }^{+}$ while anticorrelated with turbidity. This result seems coherent as higher efficiency of solidliquid separation should tend to remove COD as suspended solids reducing turbidity, 
while increasing the concentration of soluble compounds. Furthermore, the utilization of coagulants and polymers in several separation techniques led to a high correlation relating separation efficiency with conductivity, with a slightly lower correlation observed between separation efficiency with alkalinity. Some commonly used coagulants are metallic salts, for instance ferric chloride or aluminum sulfate, which react with bicarbonate in order to form metallic hydroxides $\left(\mathrm{Fe}(\mathrm{OH})_{3}, \mathrm{Al}(\mathrm{OH})_{3}\right)$ [9].

Other high correlations observed were between alkalinity and IC, dissolved TOC, TKN (total, suspended, colloids and dissolved), dissolved organic $\mathrm{N}, \mathrm{NH}_{4}{ }^{+}$and conductivity. It seems a trivial correlation as IC consist of a major part of alkalinity (carbonates) as well as ammoniacal nitrogen, which is greatly responsible for digestate buffering capacity. Meanwhile, alkalinity was anticorrelated with $\mathrm{C} / \mathrm{N}$ in liquid fractions of digestates, possibly because of high ammoniacal nitrogen contributing to alkalinity and, thus, to low $\mathrm{C} / \mathrm{N}$. Besides, the correlations between all nitrogen measurements (TKN, dissolved organic N, $\mathrm{NH}_{4}{ }^{+}, \mathrm{C} / \mathrm{N}$ ) were also observed, together with their correlation with conductivity.

$\mathrm{BOD}_{5}$ is positively correlated with $\mathrm{BOD}_{21}(\mathrm{r}=0.85)$. Indeed, $\mathrm{BOD}_{21}$ comprises the $\mathrm{BOD}_{5}$ parameter; which justifies the relation between these parameters. In this study, the mean value of $\mathrm{BOD}_{5} / \mathrm{BOD}_{21}$ obtained was $0.43 \pm 0.12$. Notably, this value is much lower than the usual ratio of $\mathrm{BOD}_{5} / \mathrm{BOD}_{21}$ from 0.6 to 0.9 observed for raw sewage [51]; $\mathrm{BOD}_{5} / \mathrm{BOD}_{21}$ could be a relevant parameter for digestate characterization.

In the range of moderate correlation coefficients $(0.5<|\mathrm{r}|<0.7)$, several correlations between parameters were identified. In relation to feedstock composition, cereal residues fraction (Cer) was shown to link to CST in consequence to small particles in liquid fractions of digestates, while energy crop residues (EnCr) appeared to be interconnected to SUVA 254 as an aromatic content indicator in the digestates. The lignin content of the energy crop residue is generally discussed in the literature; explaining the low methane potential of these compounds. SUVA 254 and CST were found to be parameters which validate the organic matter residual content in digestates. Hermann et al. [52] and Dandikas et al. [53] investigated various crop silages and grassland, respectively; both have concluded that the SUVA 254 increase after anaerobic digestion process with limited biomethane potential $(\mathrm{BMP})$ is due to presence of lignin found in these feedstocks.

In this study, some parameters were more signified for correlation with various parameters. Turbidity, conductivity, $\mathrm{SUVA}_{254}$ and CST were the utmost common parameters which also justified the main part of digestates' characteristics. From a practical perspective, apart from $\mathrm{SUVA}_{254}$, these measurements are simple to conduct with shorter time required to obtain results of the liquid fractions of digestates' characteristics (e.g., conductivity measurement to obtain separation efficiency, alkalinity, IC, C/N, TKN total, TKN colloids, TKN dissolved, $\mathrm{N}$ organic dissolved and $\mathrm{NH}_{4}{ }^{+}$of liquid fractions of digestates).

\subsection{Multivariate Analysis via Principal Component Analysis (PCA): Impact of Solid-Liquid Separation Techniques}

A PCA was carried out including on all the 42 variables (center-scaled) of all the 30 digestates as shown in Figure 1. The first PCA component describes $32 \%$ of the variance while the second component describes $16 \%$. Considering the high number (42) of very diverse variables included in the analysis, a description of almost $50 \%$ of total variance with only two components highlights the power of PCA.

The PCA in Figure 1 shows that the liquid fractions of digestates could be categorized by the types of solid-liquid separation techniques applied. Sequentially, calculation on separation efficiency or removal efficiency (R) based on Equation (1) allowed us to evaluate the influence of solid-liquid separators and the results are presented in Figure 2.

Remarkably, the screw press, vibrating screen and rotary drum were classified in the group of solid-liquid separation with low separation efficiency (with values $\leq 44 \%$ ). In contrast, the centrifuge and other types of solid-liquid separators with the addition of either coagulants, flocculents or polymers were classified as high efficiency separators with 44 to $93 \%$ separation efficiency. This study confirmed the low separation efficiencies of the screw press ( $<30 \%$ efficiencies) compared to the centrifuge (from 33 to $69 \%$ efficiencies) 
obtained previously in a study by Moller [54]. A meta-analysis study with over 60 fullscale separators resulted in a similar observation where, based on the same indicator, two efficiency groups could be observed and similarly linked to feedstock [5].

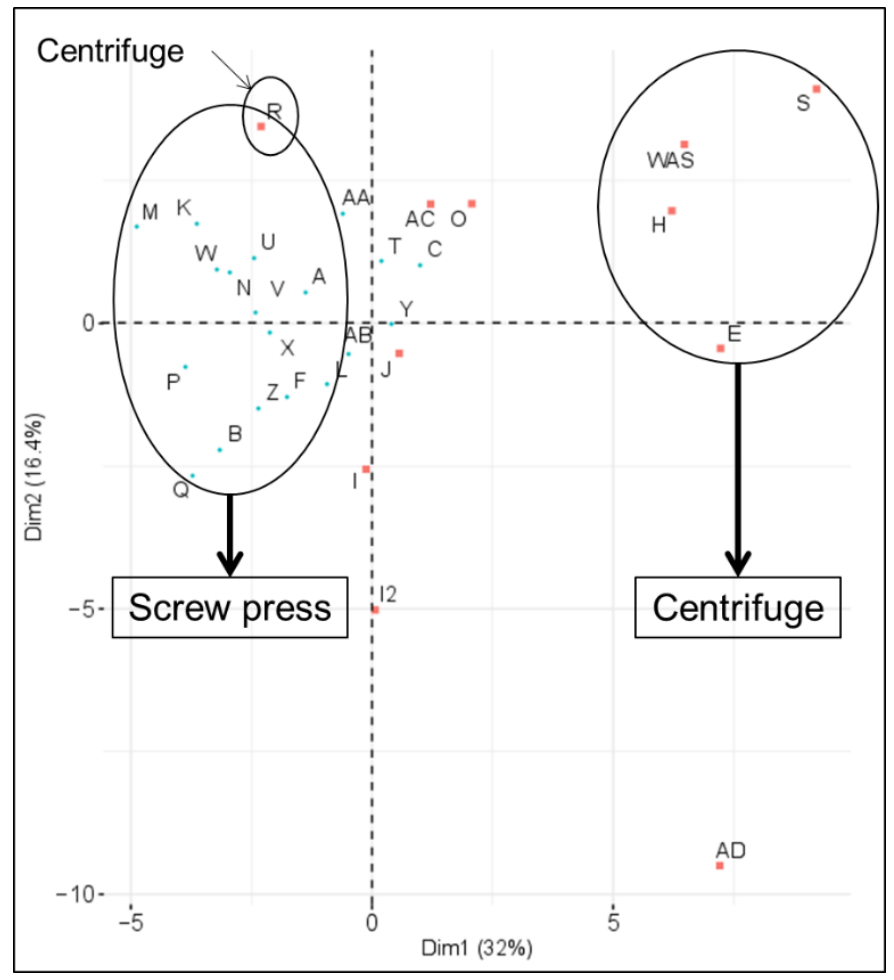

Figure 1. PCA Individuals: Impact of solid-liquid separation.

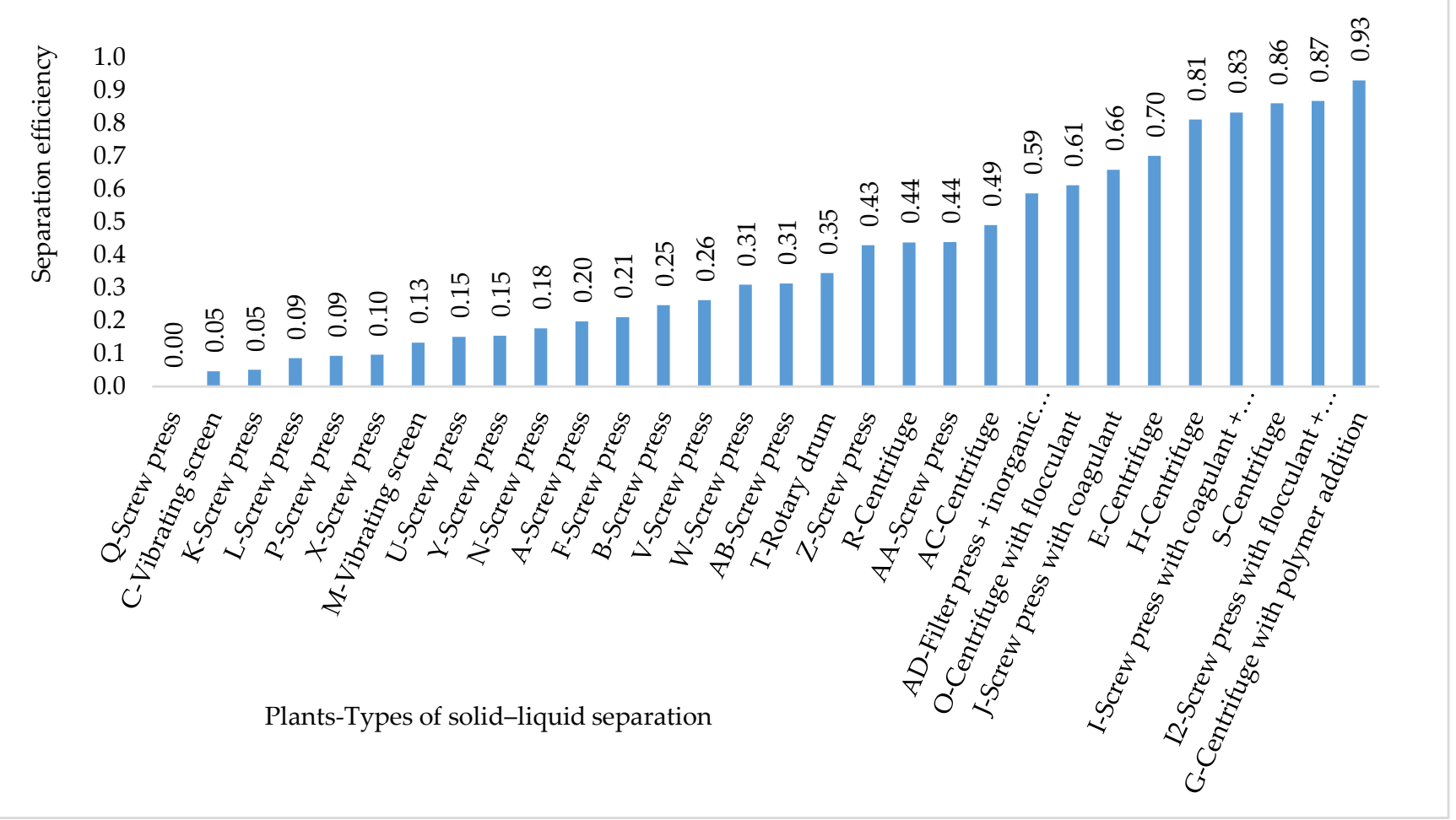

Figure 2. Separation efficiency vs. types of solid-liquid separators used for plants. 
Even though digestate $\mathrm{R}$ was separated by centrifugation, it had a low separation efficiency of $44 \%$ only and, hence, it belonged to the group with low efficiency separation. This may possibly be owing to the inefficient centrifuge applied for digestate separation.

The liquid fractions of digestates I, I2 and J from high efficiency separators, each with 83,87 and $66 \%$ separation efficiency, respectively (Figure 2), were, however, near to low efficiency solid-liquid separation group (Figure 1). This may possibly be due to the fact that I, I2 and J were originated from T-PF anaerobic digesters operated at high total solid content (dry-AD) where the organic matter was not completely digested during the process. As a result, the amounts of undigested organic matter remained high although an efficient solid-liquid separator was used, resulting in liquid fractions of digestates with high VS/TS, COD total/TS, COD suspended/TS and COD colloids/TS.

In contrast, $\mathrm{T}$ (originated from pig slurry, corn silage and fats), $\mathrm{C}$ (originated from fruits and vegetable waste) and $\mathrm{Y}$ (originated from biowaste, cereals and fats) from low efficiency solid-liquid separators (separation efficiency of 35, 5 and 15\%, respectively) (Figure 2), however, were near to the cluster of solid-liquid separators with high performance, as shown in PCA in Figure 1. This may possibly be explained by the origins of the easily biodegradable substrates, resulting in lower residual organic matters in the liquid fractions of digestates.

The loading scores of measured parameters of Dimension 1 of the PCA is shown in Figure 3. Positive values were correlated to high efficiency separation, which is correlated to dissolved inorganic, alkalinity, ions with significant parameters of conductivity, TKN dissolved, TKN total, $\mathrm{IC}, \mathrm{NH}_{4}{ }^{+}$, dissolved organic nitrogen and alkalinity with value $>0.8$. On the contrary, negative values were correlated to low efficiency which is correlated to organic matter and solids. The significant parameters observed were dissolved $\mathrm{C} / \mathrm{N}$, turbidity, COD suspended, VS liquid and CST with values $<-0.6$. A meta-analysis study on digestate quality with a database containing about 150 raw digestates, solid and liquid fractions resulted in a very similar observation [8]. This result can be associated with the fact that low efficiency separators such as screw presses are mainly applied to fibrous inputs which are mostly poorer in $\mathrm{N}$ content while results in digestates with greater recalcitrant organic matter (higher $C$ content). At the same time, high performance separation equipment such as centrifuges are widely applied to non-fibrous inputs such as pig slurry and biowaste which are commonly $\mathrm{N}$-rich and more biodegradable.

\subsection{Influence of Feedstock Composition on Digestate Characteristics}

Hierarchical Clustering Analysis (HCA) was carried out to evaluate the influence of the feedstock composition. Sequentially, to remove the influence of solid-liquid separators, HCA was separately implemented according to high performance of solid-liquid separators (centrifuge and other types of separators with addition of coagulant, flocculent or polymer) and low performance solid-liquid separators (screw press, vibrating screen and rotary drum), as shown in Figure 4. In high performance separation group (left side of Figure 4), two major clusters of liquid fractions can be identified based on AD feedstock. The first cluster was primarily from sewage sludge codigested with Mnr (pig manure), FOG and AFW. Meanwhile, the second cluster identified was primarily originated from agricultural and industrial wastes. This cluster can then be divided by two subclusters; liquid phase anaerobic digestion (L-AD) from mesophilic CSTR and solid-state anaerobic digestion (SS-AD) from T-PF reactor. From the observation in the subgroup of L-AD, the influence of higher manure proportion formed the group apart the other major group of L-AD primarily from sewage sludge.

The clustering of low performance solid-liquid separation was less marked regarding AD feedstock but can also be separated into two major clusters. The first cluster was predominantly originating either from pig manure, FOG or AFW. Meanwhile, the origin of the second cluster was from the codigestion of cow manure and diverse agricultural and industrial wastes, including sewage sludge.

Figure 5 plotted below aims to analyze the influence of substrates' composition on the 
liquid fractions of digestates, specifically on final COD concentration. From the observation, a correlation $R^{2}=0.53$ ( $p$ value $\left.<0.1\right)$ exists between cow manure percentage in the feedstock and the COD concentration. Having a larger sample size made it possible to confirm the observations made by Akhiar et al. [13] with 11 digestates, which also confirmed a study by Ganesh et al. [55] where the increase of cow manure proportions in the feed led to higher COD concentration in the liquid fractions of digestates. It was also observed that higher energy crops' proportion in the feedstock may possibly influence the COD concentration in the liquid fractions of digestates with $\mathrm{R}^{2}=0.24(p$ value $<0.1)$ (Figure 5). This correlation is not robust and should be confirmed in further work.

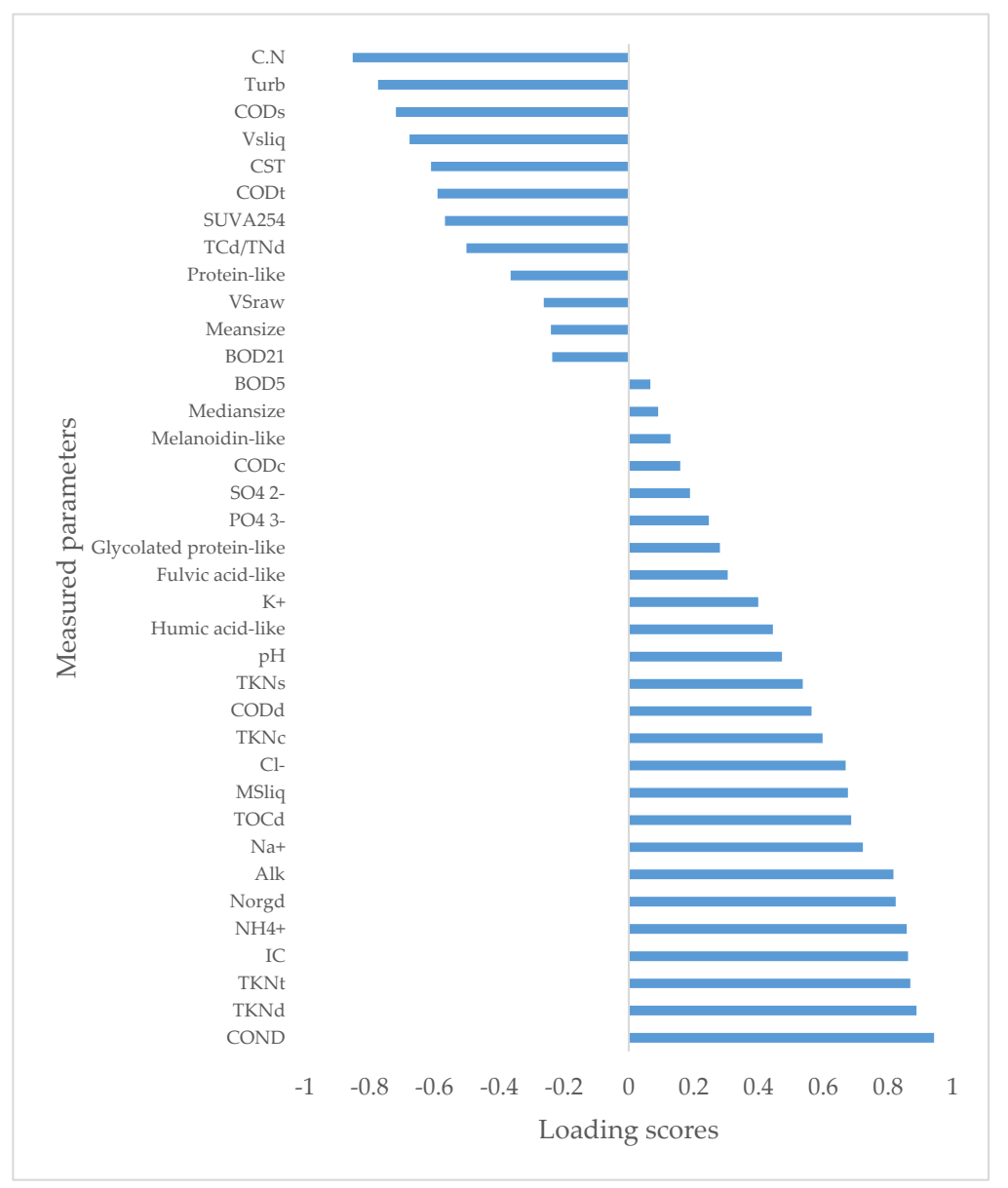

Figure 3. Loading scores of measured parameters of Dimension 1.

3.4. Influence of Anaerobic Digestion Operating Parameters on Digestate Characteristics: Impact of HRT on Liquid Fractions of Digestates

In this study, the parameter with the highest correlation to HRT observed was $\mathrm{SUVA}_{254}$ with $\mathrm{R}=0.52(p$ value $<0.01)$ as presented in Figure 6a. The $\mathrm{SUVA}_{254}$ is a common indicator of the aromatic content of the organic matter in water and wastewater. When an anaerobic digester is set to a longer HRT, it could be presumed that independently of the substrates at the input, the degree of humification in the digester rises proportionally with $\mathrm{SUVA}_{254}$. This correlation was previously studied by Zheng et al. [39] with a variety of biodegradable substrates confirming proportional correlation between $\mathrm{SUVA}_{254}$ and degradation time. Besides, the final $\mathrm{SUVA}_{254}$ was also observed to vary depending on the types of substrates. Given that HRT correspondingly depends on the types of substrates, an indirect relation between $\mathrm{SUVA}_{254}$ and the characteristics of the substrates can be presumed.

The degree of humification should also be represented by the measurements of fluorimetry, describing humic acidlike area. Figure $6 \mathrm{~b}$ intended to examine $\mathrm{SUVA}_{254}$ and its relation with 3D fluorescence spectrum zones; however, no correlation between fluorimetry 
fractions (in particular humic acidlike area) of digestates and $\mathrm{SUVA}_{254}$ was observed. This was previously shown by Yang et al. [56] and Bioroza et al. [57] for the organic matter in water and drinking water, respectively. These two indicators, $\mathrm{SUVA}_{254}$ and $3 \mathrm{D}$ fluorimetry, signify the humification intensity but from different molecules; both indicators are then incommutable.

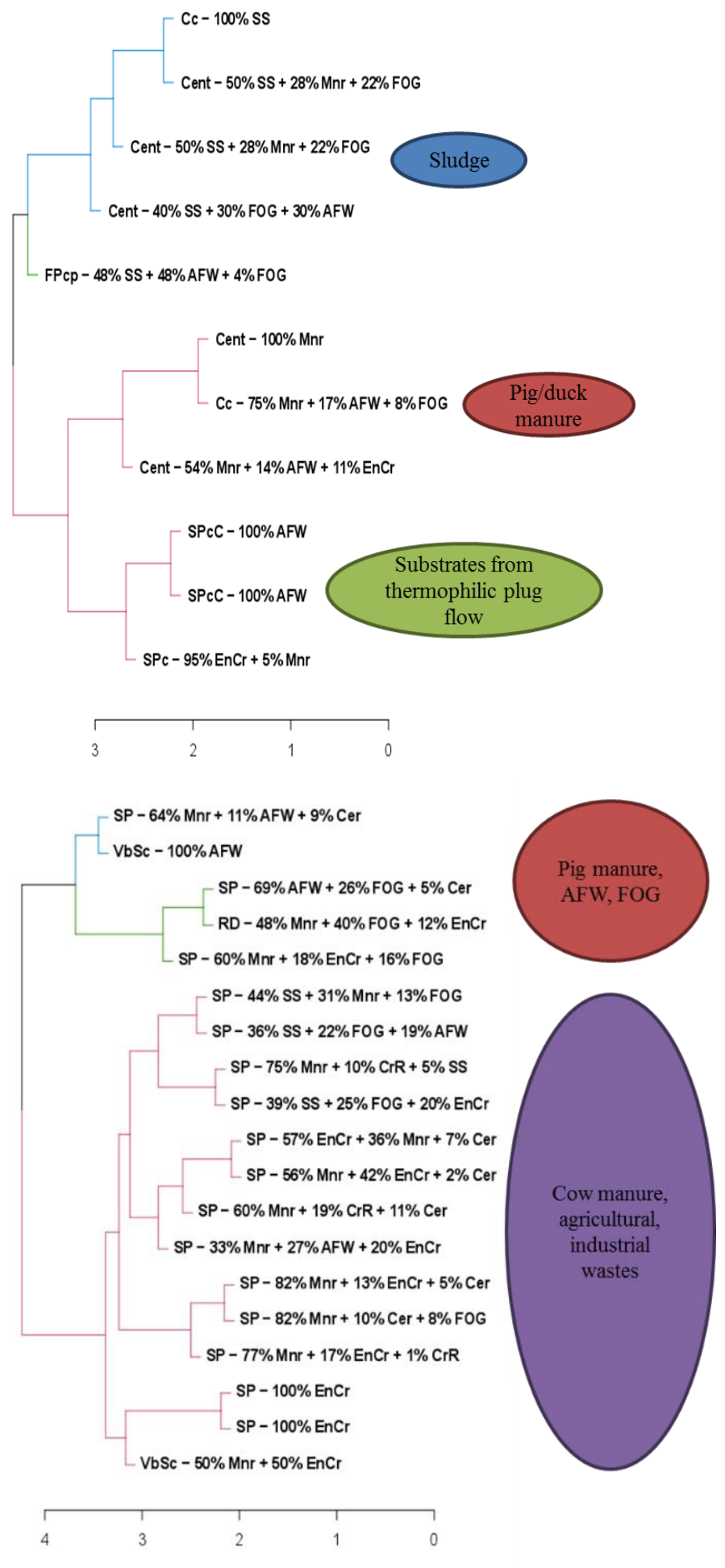

Figure 4. Clustering of feedstocks (Left: High performance solid-liquid-solid separation, Right: Low performance solid-liquid separation). 

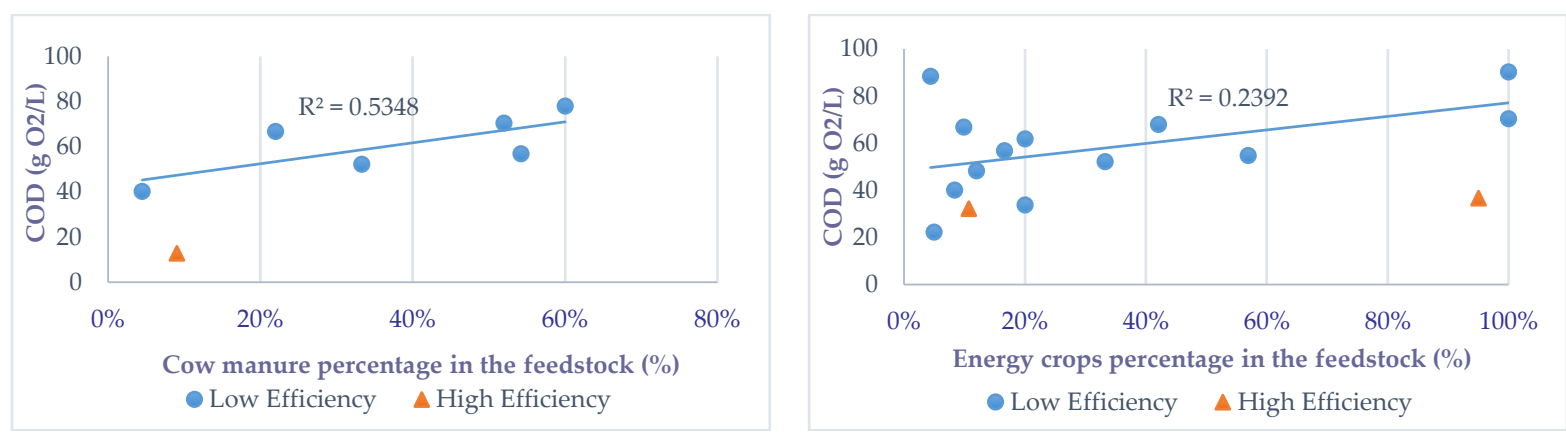

Figure 5. (Left) COD in the liquid fraction vs cow manure percentage and (right) COD in the liquid fraction vs energy crops percentage in the feedstock.

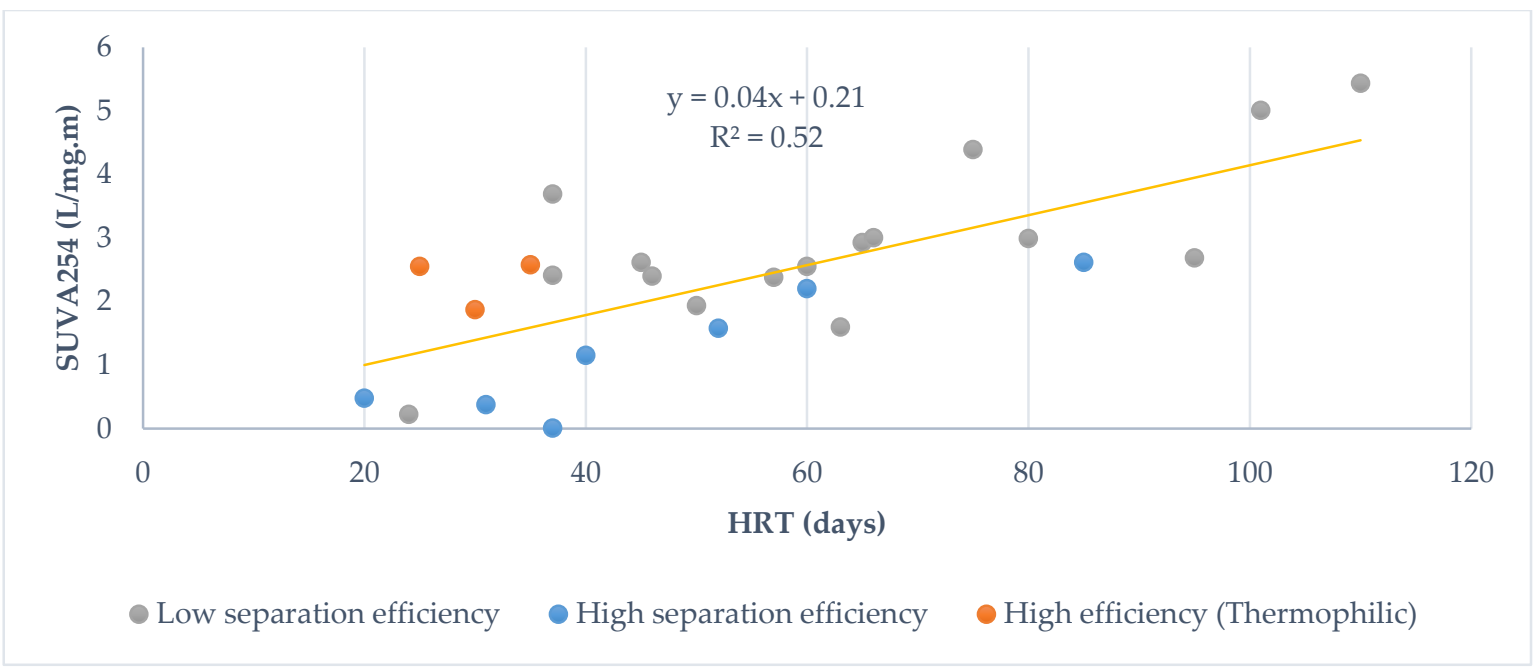

(a)

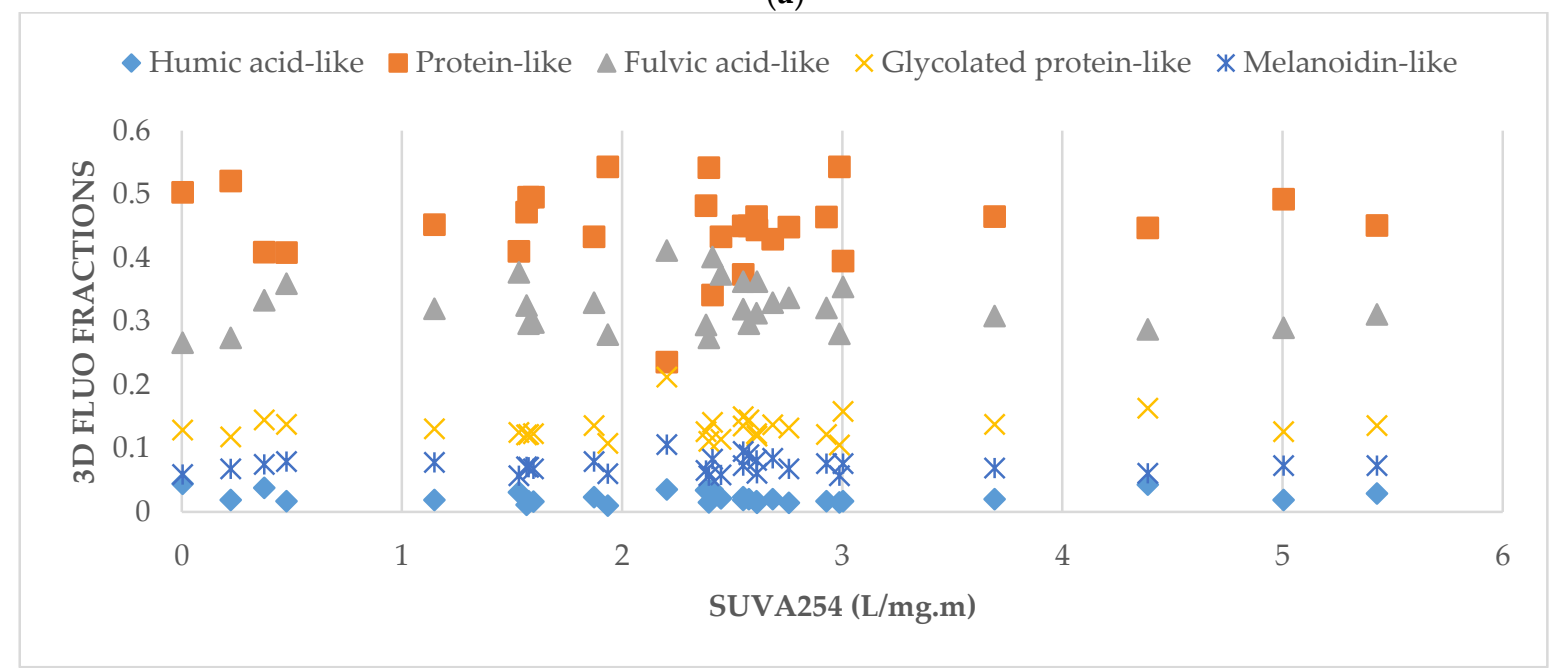

(b)

Figure 6. (a) $\mathrm{SUVA}_{254}(\mathrm{~L} / \mathrm{mg} \cdot \mathrm{m})$ in the liquid fraction vs HRT (days) and (b) 3D fluorescence spectrum zones vs SUVA 254 $(\mathrm{L} / \mathrm{mg} \cdot \mathrm{m})$.

\subsection{Outcome of the Work}

The separation technique and anaerobic digestion feedstock were identified as the major drivers of the remaining organic matter characteristics in the liquid fractions of 
digestates, with the separation technique being usually selected according to the feedstock. This study allowed us to define different categories of liquid fractions of digestates:

- Digestates from sewage sludge, pig manure and from thermophilic plug-flow reactor whose phase separation is carried out by high efficiency techniques (e.g., centrifugation, and other techniques using flocculant or coagulants) and

- Digestates from agricultural fibrous feedstocks which are processed by low efficiency technique processes (e.g., screw presses, vibrating screens and rotary drums). In particular, cow manure content in the feedstock was found to have high impact on the remaining COD in the liquid fraction of digestate.

These categories would set reference compositions in relation to process conditions and will support better knowledge of the liquid fractions of digestates. In addition, this work can be useful for example to practitioners when designing appropriate post-treatment of the digestates. It can also be useful to identify new solutions of the post-treatment of digestate by maximizing its utilization towards achieving circular economy.

\section{Conclusions}

The combined effect of the solid-liquid separation technique and anaerobic digestion feedstock were identified as the major drivers of the remaining organic matter characteristics in the liquid fractions of digestates. Two major clusters were identified in this study: (1) high-performance solid-liquid separators such as centrifuge and other separation systems with addition of coagulant, flocculent or polymer (separation efficiency from 44 to $93 \%$ ) are mainly applied to digestates from sewage sludge, pig manure and from plug-flow thermophilic processes; (2) low-performance solid-liquid separators such as screw presses, vibrating screens and rotary drums (separation efficiency not more than $44 \%$ ) are commonly applied to fibrous digestates; in this case, increasing the percentage of cow manure or energy crops in the feedstocks contents' were identified as the contributing factors to the increase in the remaining organic compounds in the liquid fractions of digestates. Notably, cow manure percentage in the feedstocks had a robust correlation with the concentration of COD in liquid fractions of digestates. Besides, amongst all the operational parameters observed, longer HRT applied to the reactor appears to have an impact to higher value of $\mathrm{SUVA}_{254}$, associated with fulvic acid compounds in dissolved matter. This indicator fits to describe the organic matter stabilization after biodegradation.

Author Contributions: Conceptualization, F.G. and M.T.; data curation, A.A. and A.B.; formal analysis, A.A., F.G. and A.B.; funding acquisition, A.H.S.; investigation, A.A. and M.T.; methodology, A.A., F.G. and A.B.; project administration, M.T. and H.C.; resources, M.T. and A.B.; software, F.G.; Supervision, M.T., A.B. and H.C.; validation, A.A., F.G. and H.C.; visualization, F.G.; writingoriginal draft, A.A., A.B.; writing-review and editing, A.A., M.T., A.B. and H.C. All authors have read and agreed to the published version of the manuscript.

Funding: This research was funded by Majlis Amanah Rakyat Malaysia and AAIBE Chair of Renewable Energy, Grant No. 201801 KETTHA.

Institutional Review Board Statement: Not applicable.

Informed Consent Statement: Not applicable.

Acknowledgments: The authors would like to express their deepest gratitude to INRAE Bio2E Facility (Bio2E, INRAE, 2018. Environmental Biotechnology and Biorefinery Facility (https: / doi. org/10.15454/1.557234103446854E12) where all experiments were conducted. The authors would also like to thank Majlis Amanah Rakyat Malaysia and AAIBE Chair of Renewable Energy, Grant No. 201801 KETTHA for the financial assistance obtained for the execution of this research. The authors are also indebted to Emilie Gout and Phillipe Sousbie for their kind support in the laboratory.

Conflicts of Interest: The authors declare no conflict of interest. The funders had no role in the design of the study; in the collection, analysis, or interpretation of data; in the writing of the manuscript, or in the decision to publish the results. 


\section{Appendix A}

Table A1. Substrate categories based on substrates at the input.

\begin{tabular}{|c|c|}
\hline Substrate Category & Substrates at the Input \\
\hline $\begin{array}{l}\text { Sewage sludge } \\
\text { (SS) }\end{array}$ & Sludge, solid sludge, liquid sludge, waste activated sludge, cheese plant sludge, contents from septic tanks (and garbage), wastewater \\
\hline $\begin{array}{l}\text { Manure } \\
\text { (Mnr) }\end{array}$ & Animal manures and slurry \\
\hline $\begin{array}{l}\text { Energy crops } \\
\quad(\text { EnCr) }\end{array}$ & Energy crops, catch crop, corn silage, grass silage, grass, energy crop silage, whole grain plants, sorghum silage, barley, rye \\
\hline $\begin{array}{l}\text { Crop residues } \\
(\mathrm{CrR})\end{array}$ & Crop residues, corn withers, sweetcorn cobs, tomato leaves, apple pomace \\
\hline $\begin{array}{l}\text { Cereal residues } \\
\quad(\text { Cer })\end{array}$ & Cereal residues, crushed grain \\
\hline $\begin{array}{l}\text { Fats, oil and grease } \\
\text { (FOG) }\end{array}$ & Fats, oil, grease \\
\hline $\begin{array}{l}\text { Agro-food wastes } \\
\quad(\text { AFW) }\end{array}$ & $\begin{array}{l}\text { Food wastes, fruit and vegetable wastes, municipal biowastes, biowastes, glucose, cattle feed residues, pet food, milk industry residues, mixture of } \\
\text { cream milk, slaughterhouse wastes, blood, glycerin, whey }\end{array}$ \\
\hline
\end{tabular}


Table A2. Total solids (TS), volatile solids (VS) and mineral solids (MS), VS/TS, pH, alkalinity, turbidity, capillary suction time (CST) and particle sizes.

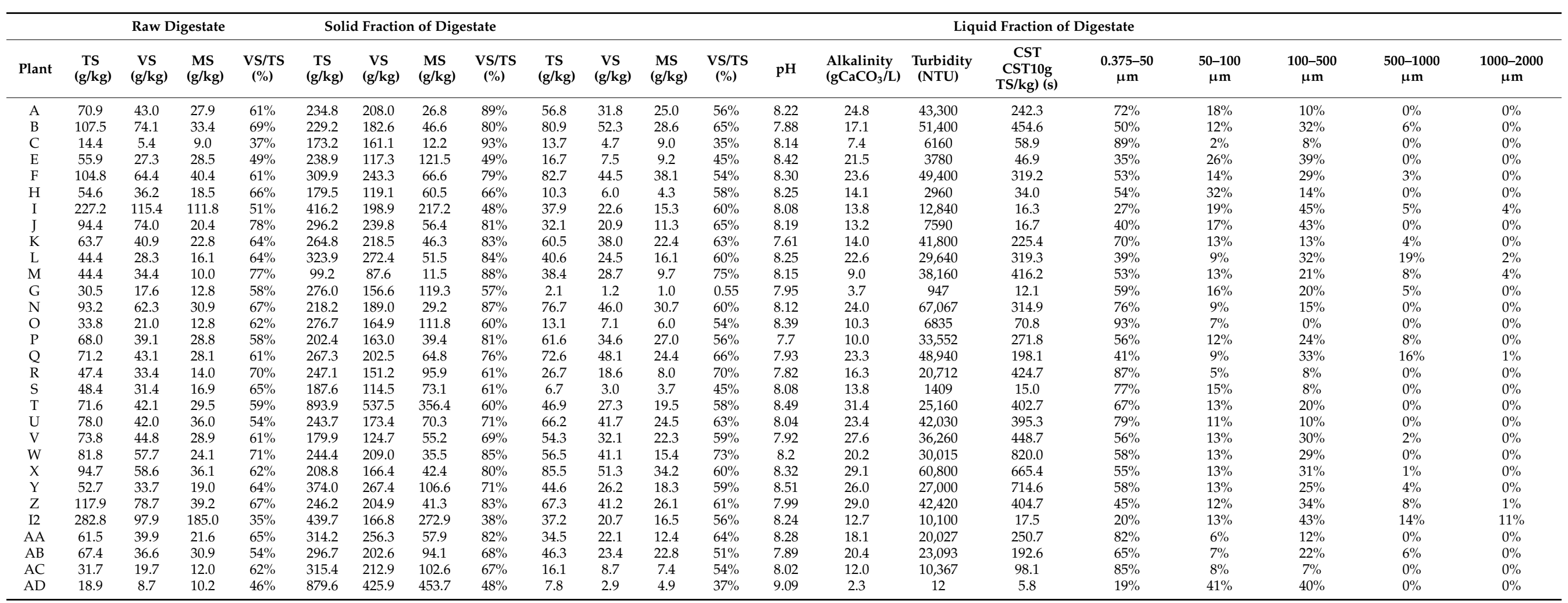




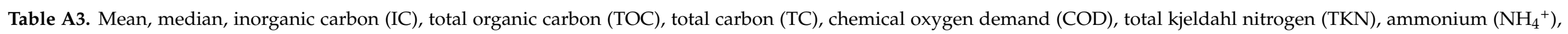
nitrogen $(\mathrm{N})$, ammonium $\left(\mathrm{NH}_{4}^{+}\right)$, carbon/nitrogen ration $(\mathrm{C} / \mathrm{N})$, sodium $\left(\mathrm{Na}^{+}\right)$, potassium $\left(\mathrm{K}^{+}\right)$, chlorine $\left(\mathrm{Cl}^{-}\right)$, phosphate $\left(\mathrm{PO}_{4}{ }^{3-}\right)$, sulfate $\left(\mathrm{SO}_{4}{ }^{2-}\right)$, conductivity $(\mathrm{COND})$.

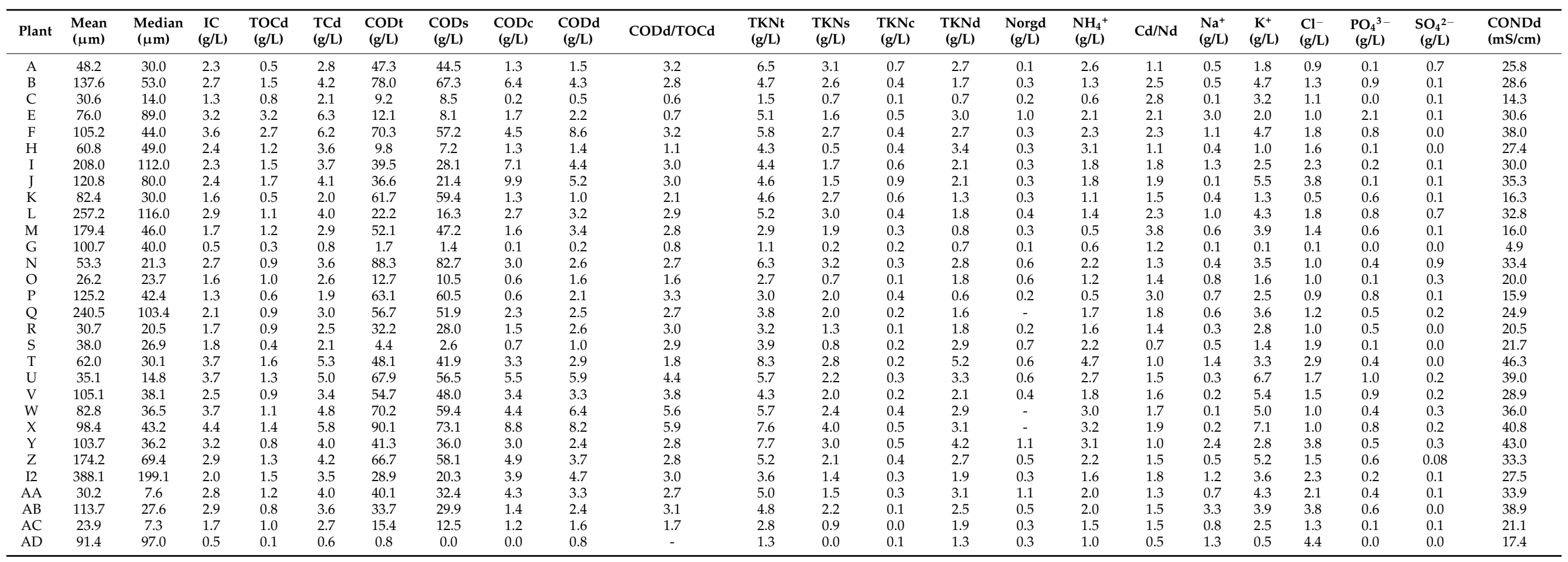

$\mathrm{t}=$ total $\mathrm{s}=$ suspended $\mathrm{c}=$ colloids, $\mathrm{d}=$ dissolved, orgd $=$ dissolved organic. 
Table A4. $\mathrm{SUVA}_{254}, \mathrm{BOD}_{5}, \mathrm{BOD}_{21}, \mathrm{BOD}_{5} / \mathrm{COD}, \mathrm{BOD}_{21} / \mathrm{COD}$, protein-like, fulvic acid-like, glycolated protein-like, melanoidin-like, humic acid-like.

\begin{tabular}{|c|c|c|c|c|c|c|c|c|c|c|}
\hline Plant & SUVA $_{254}$ & $\begin{array}{c}\text { BOD }_{5} \\
(g / L)\end{array}$ & $\begin{array}{c}\mathrm{BOD}_{21} \\
(\mathrm{~g} / \mathrm{L})\end{array}$ & $\mathrm{BOD}_{5} / \mathrm{COD}$ & $\mathrm{BOD}_{21} / \mathrm{COD}$ & Protein-Like & Fulvic Acid-Like & Glycolated Protein-Like & Melanoidin-Like & Humic Acid-Like \\
\hline A & 1.6 & 7.4 & 23.2 & 0.2 & 0.5 & $47 \%$ & $33 \%$ & $12 \%$ & $7 \%$ & $1 \%$ \\
\hline $\mathrm{B}$ & 2.6 & 5.6 & 12.9 & 0.1 & 0.2 & $37 \%$ & $36 \%$ & $15 \%$ & $10 \%$ & $2 \%$ \\
\hline $\mathrm{C}$ & 0.2 & 1.7 & 3.1 & 0.2 & 0.3 & $52 \%$ & $27 \%$ & $12 \%$ & $7 \%$ & $2 \%$ \\
\hline $\mathrm{E}$ & 0.0 & 1.9 & 4.3 & 0.2 & 0.4 & $50 \%$ & $27 \%$ & $13 \%$ & $6 \%$ & $4 \%$ \\
\hline $\mathrm{F}$ & 1.6 & 3.7 & 9.8 & 0.1 & 0.1 & $50 \%$ & $30 \%$ & $12 \%$ & $7 \%$ & $2 \%$ \\
\hline $\mathrm{H}$ & 0.4 & 3.7 & 5.3 & 0.4 & 0.5 & $41 \%$ & $33 \%$ & $15 \%$ & $7 \%$ & $4 \%$ \\
\hline I & 1.9 & 7.3 & 18.1 & 0.2 & 0.5 & $43 \%$ & $33 \%$ & $14 \%$ & $8 \%$ & $2 \%$ \\
\hline $\mathrm{K}$ & 1.9 & 9.4 & 32.2 & 0.2 & 0.5 & $54 \%$ & $28 \%$ & $11 \%$ & $6 \%$ & $1 \%$ \\
\hline $\mathrm{L}$ & 2.4 & 4.8 & 11.1 & 0.2 & 0.5 & $54 \%$ & $27 \%$ & $11 \%$ & $6 \%$ & $2 \%$ \\
\hline $\mathrm{M}$ & 3.0 & 5.0 & 14.3 & 0.1 & 0.3 & $54 \%$ & $28 \%$ & $11 \%$ & $6 \%$ & $2 \%$ \\
\hline G & 0.5 & 0.4 & 1.0 & 0.2 & 0.6 & $41 \%$ & $36 \%$ & $14 \%$ & $8 \%$ & $2 \%$ \\
\hline $\mathrm{N}$ & 2.7 & 13.9 & 42.6 & 0.2 & 0.5 & $43 \%$ & $33 \%$ & $14 \%$ & $8 \%$ & $2 \%$ \\
\hline $\mathrm{O}$ & 1.6 & 0.3 & 1.3 & 0.0 & 0.1 & $50 \%$ & $30 \%$ & $12 \%$ & $7 \%$ & $2 \%$ \\
\hline $\mathrm{P}$ & 2.9 & 3.7 & 27.8 & 0.1 & 0.4 & $46 \%$ & $32 \%$ & $12 \%$ & $8 \%$ & $2 \%$ \\
\hline $\mathrm{Q}$ & 2.8 & 7.4 & 18.2 & 0.1 & 0.3 & $45 \%$ & $34 \%$ & $13 \%$ & $7 \%$ & $1 \%$ \\
\hline$\hat{\mathrm{R}}$ & 2.6 & 9.6 & 20.7 & 0.3 & 0.6 & $44 \%$ & $36 \%$ & $12 \%$ & $6 \%$ & $1 \%$ \\
\hline $\mathrm{T}$ & 1.5 & 13.1 & 28.3 & 0.3 & 0.6 & $41 \%$ & $38 \%$ & $12 \%$ & $6 \%$ & $3 \%$ \\
\hline $\mathrm{U}$ & 5.0 & 13.0 & 30.6 & 0.2 & 0.5 & $49 \%$ & $29 \%$ & $13 \%$ & $7 \%$ & $2 \%$ \\
\hline $\mathrm{V}$ & 3.7 & 12.0 & 28.2 & 0.2 & 0.5 & $46 \%$ & $31 \%$ & $14 \%$ & $7 \%$ & $2 \%$ \\
\hline $\mathrm{W}$ & 5.4 & 16.6 & 35.6 & 0.2 & 0.5 & $45 \%$ & $31 \%$ & $14 \%$ & $7 \%$ & $3 \%$ \\
\hline$x$ & 4.4 & 30.1 & 54.2 & 0.3 & 0.6 & $45 \%$ & $29 \%$ & $16 \%$ & $6 \%$ & $4 \%$ \\
\hline $\mathrm{Y}$ & 2.4 & 16.2 & 26.0 & 0.4 & 0.6 & $34 \%$ & $40 \%$ & $14 \%$ & $8 \%$ & $3 \%$ \\
\hline $\mathrm{Z}$ & 3.0 & 14.5 & 35.0 & 0.2 & 0.5 & $39 \%$ & $35 \%$ & $16 \%$ & $8 \%$ & $2 \%$ \\
\hline $\mathrm{I} 2$ & 2.6 & 9.6 & 17.8 & 0.3 & 0.6 & $45 \%$ & $32 \%$ & $14 \%$ & $7 \%$ & $2 \%$ \\
\hline AA & 2.6 & 7.2 & 17.6 & 0.2 & 0.4 & $46 \%$ & $31 \%$ & $12 \%$ & $8 \%$ & $2 \%$ \\
\hline $\mathrm{AB}$ & 2.4 & 11.4 & 25.4 & 0.3 & 0.8 & $48 \%$ & $29 \%$ & $13 \%$ & $6 \%$ & $3 \%$ \\
\hline $\mathrm{AC}$ & 1.1 & 3.7 & 8.0 & 0.2 & 0.5 & $45 \%$ & $32 \%$ & $13 \%$ & $8 \%$ & $2 \%$ \\
\hline $\mathrm{AD}$ & 2.2 & 0.1 & 0.4 & 0.1 & 0.4 & $24 \%$ & $41 \%$ & $21 \%$ & $11 \%$ & $3 \%$ \\
\hline
\end{tabular}




\section{References}

1. European Biogas Association (EBA). Statistical Report of the European Biogas Association 2018; EBA: Brussels, Belgium, 2018.

2. Akhiar, A.; Ahmad Zamri, M.F.M.; Torrijos, M.; Shamsuddin, A.H.; Battimelli, A.; Roslan, E.; Mohd Marzuki, M.H.; Carrere, H. Anaerobic digestion industries progress throughout the world. IOP Conf. Ser. Earth Environ. Sci. 2020, 476, 012074. [CrossRef]

3. Battista, F.; Frison, N.; Bolzonella, D. Energy and Nutrients' Recovery in Anaerobic Digestion of Agricultural Biomass: An Italian Perspective for Future Applications. Energies 2019, 12, 3287. [CrossRef]

4. Herbes, C.; Dahlin, J.; Kurz, P. Consumer Willingness To Pay for Proenvironmental Attributes of Biogas Digestate-Based Potting Soil. Sustainability 2020, 12, 6405. [CrossRef]

5. Guilayn, F.; Jimenez, J.; Martel, J.-L.; Rouez, M.; Crest, M.; Patureau, D. First fertilizing-value typology of digestates: A decision-making tool for regulation. Waste Manag. 2019, 86, 67-79. [CrossRef] [PubMed]

6. Szymańska, M.; Szara, E.; Sosulski, T.; Wąs, A.; Van Pruissen, G.W.P.; Cornelissen, R.L.; Borowik, M.; Konkol, M. A Bio-Refinery Concept for N and P Recovery-A Chance for Biogas Plant Development. Energies 2019, 12, 155. [CrossRef]

7. Maurer, C.; Seiler-Petzold, J.; Schulz, R.; Müller, J. Short-Term Nitrogen Uptake of Barley from Differently Processed Biogas Digestate in Pot Experiments. Energies 2019, 12, 696. [CrossRef]

8. Guilayn, F.; Jimenez, J.; Rouez, M.; Crest, M.; Patureau, D. Digestate mechanical separation: Efficiency profiles based on anaerobic digestion feedstock and equipment choice. Bioresour. Technol. 2019, 274, 180-189. [CrossRef]

9. Drosg, B.; Fuchs, W.; Al Seadi, T.; Madsen, M.; Linke, B. Nutrient Recovery by Biogas Digestate Processing; 2015. Available online: http:/ / task37.ieabioenergy.com/files/daten-redaktion/download/Technical\%20Brochures/NUTRIENT_RECOVERY_ RZ_web1.pdf (accessed on 23 November 2020).

10. Bauer, A.; Mayr, H.; Hopfner-Sixt, K.; Amon, T. Detailed monitoring of two biogas plants and mechanical solid-liquid separation of fermentation residues. J. Biotechnol. 2009, 142, 56-63. [CrossRef]

11. Rehl, T.; Müller, J. Life cycle assessment of biogas digestate processing technologies. Resour. Conserv. Recycl. 2011, 56, 92-104. [CrossRef]

12. Tambone, F.; Orzi, V.; D'Imporzano, G.; Adani, F. Solid and liquid fractionation of digestate: Mass balance, chemical characterization, and agronomic and environmental value. Bioresour. Technol. 2017, 243, 1251-1256. [CrossRef]

13. Akhiar, A.; Torrijos, M.; Battimelli, A.; Carrère, H. Comprehensive characterization of the liquid fraction of digestates from full-scale anaerobic co-digestion. Waste Manag. 2017, 59, 118-128. [CrossRef] [PubMed]

14. Xia, A.; Murphy, J.D. Microalgal Cultivation in Treating Liquid Digestate from Biogas Systems. Trends Biotechnol. 2016, 34, 264-275. [CrossRef] [PubMed]

15. Baldi, M.; Collivignarelli, M.; Abbà, A.; Benigna, I. The Valorization of Ammonia in Manure Digestate by Means of Alternative Stripping Reactors. Sustainability 2018, 10, 3073. [CrossRef]

16. Valentinuzzi, F.; Cavani, L.; Porfido, C.; Terzano, R.; Pii, Y.; Cesco, S.; Marzadori, C.; Mimmo, T. The fertilising potential of manure-based biogas fermentation residues: Pelleted vs. liquid digestate. Heliyon 2020, 6, e03325. [CrossRef]

17. Guilayn, F.; Rouez, M.; Crest, M.; Patureau, D.; Jimenez, J. Valorization of digestates from urban or centralized biogas plants: A critical review. Rev. Environ. Sci. Biotechnol. 2020, 19, 419-462. [CrossRef]

18. Tao, W.; Fattah, K.P.; Huchzermeier, M.P. Struvite recovery from anaerobically digested dairy manure: A review of application potential and hindrances. J. Environ. Manag. 2016, 169, 46-57. [CrossRef]

19. Szymańska, M.; Sosulski, T.; Szara, E.; Wąs, A.; Sulewski, P.; van Pruissen, G.W.P.; Cornelissen, R.L. Ammonium Sulphate from a Bio-Refinery System as a Fertilizer-Agronomic and Economic Effectiveness on the Farm Scale. Energies 2019, 12, 4721. [CrossRef]

20. Folino, A.; Zema, D.A.; Calabrò, P.S. Environmental and Economic Sustainability of Swine Wastewater Treatments Using Ammonia Stripping and Anaerobic Digestion: A Short Review. Sustainability 2020, 12, 4971. [CrossRef]

21. Szymańska, M.; Szara, E.; Wąs, A.; Sosulski, T.; van Pruissen, G.; Cornelissen, R. Struvite-An Innovative Fertilizer from Anaerobic Digestate Produced in a Bio-Refinery. Energies 2019, 12, 296. [CrossRef]

22. Gienau, T.; Ehrmanntraut, A.; Kraume, M.; Rosenberger, S. Influence of Ozone Treatment on Ultrafiltration Performance and Nutrient Flow in a Membrane Based Nutrient Recovery Process from Anaerobic Digestate. Membranes 2020, 10, 64. [CrossRef]

23. Świątczak, P.; Cydzik-Kwiatkowska, A.; Zielińska, M. Treatment of Liquid Phase of Digestate from Agricultural Biogas Plant in a System with Aerobic Granules and Ultrafiltration. Water 2019, 11, 104. [CrossRef]

24. Myllymäki, P.; Pesonen, J.; Romar, H.; Hu, T.; Tynjälä, P.; Lassi, U. The Use of Ca- and Mg-Rich Fly Ash as a Chemical Precipitant in the Simultaneous Removal of Nitrogen and Phosphorus-Recycling and Reuse. Recycling 2019, 4, 14. [CrossRef]

25. Krzemińska, I.; Oleszek, M.; Wiącek, D. Liquid Anaerobic Digestate as a Source of Nutrients for Lipid and Fatty Acid Accumulation by Auxenochlorella Protothecoides. Molecules 2019, 24, 3582. [CrossRef] [PubMed]

26. Kisielewska, M.; Zieliński, M.; Dębowski, M.; Kazimierowicz, J.; Romanowska-Duda, Z.; Dudek, M. Effectiveness of Scenedesmus sp. Biomass Grow and Nutrients Removal from Liquid Phase of Digestates. Energies 2020, 13, 1432. [CrossRef]

27. Jimenez, R.; Markou, G.; Tayibi, S.; Barakat, A.; Chapsal, C.; Monlau, F. Production of Microalgal Slow-Release Fertilizer by Valorizing Liquid Agricultural Digestate: Growth Experiments with Tomatoes. Appl. Sci. 2020, 10, 3890. [CrossRef]

28. Akhiar, A.; Zamri, M.F.M.A.; Torrijos, M.; Battimelli, A.; Roslan, E.; Hanif, M.; Shamsuddin, A.H.; Carrere, H. Current Technology on Nutrients Removal, Recovery and Reuse from Liquid Fraction of Digestate. TEST Eng. Manag. 2019, 81, $5861-5868$.

29. Theuerl, S.; Herrmann, C.; Heiermann, M.; Grundmann, P.; Landwehr, N.; Kreidenweis, U.; Prochnow, A. The Future Agricultural Biogas Plant in Germany: A Vision. Energies 2019, 12, 396. [CrossRef] 
30. Piwowar, A. Agricultural Biogas-An Important Element in the Circular and Low-Carbon Development in Poland. Energies 2020, 13, 1733. [CrossRef]

31. Barros, M.V.; Salvador, R.; de Francisco, A.C.; Piekarski, C.M. Mapping of research lines on circular economy practices in agriculture: From waste to energy. Renew. Sustain. Energy Rev. 2020, 131, 109958. [CrossRef]

32. Molina-Moreno, V.; Leyva-Díaz, J.; Llorens-Montes, F.; Cortés-García, F. Design of Indicators of Circular Economy as Instruments for the Evaluation of Sustainability and Efficiency in Wastewater from Pig Farming Industry. Water 2017, 9, 653. [CrossRef]

33. Muradin, M.; Joachimiak-Lechman, K.; Foltynowicz, Z. Evaluation of Eco-Efficiency of Two Alternative Agricultural Biogas Plants. Appl. Sci. 2018, 8, 2083. [CrossRef]

34. Vilardi, G.; Bassano, C.; Deiana, P.; Verdone, N. Exergy and energy analysis of three biogas upgrading processes. Energy Convers. Manag. 2020, 224, 113323. [CrossRef]

35. Ziyang, L.; Youcai, Z. Size-fractionation and characterization of refuse landfill leachate by sequential filtration using membranes with varied porosity. J. Hazard. Mater. 2007, 147, 257-264. [CrossRef]

36. American Public Health Association. Standard Methods for the Examination of Water and Wastewater, 22nd ed.; Rice, E.W., Baird, R.B., Eaton, A.D., Clesceri, L.S., Eds.; American Public Health Association: Washington, DC, USA, 2012.

37. Battimelli, A.; Loisel, D.; Garcia-Bernet, D.; Carrere, H.; Delgenes, J.-P. Combined ozone pretreatment and biological processes for removal of colored and biorefractory compounds in wastewater from molasses fermentation industries. J. Chem. Technol. Biotechnol. 2010, 85, 968-975. [CrossRef]

38. Uggetti, E.; Sialve, B.; Latrille, E.; Steyer, J.-P. Anaerobic digestate as substrate for microalgae culture: The role of ammonium concentration on the microalgae productivity. Bioresour. Technol. 2014, 152, 437-443. [CrossRef]

39. Zheng, W.; Lü, F.; Phoungthong, K.; He, P. Relationship between anaerobic digestion of biodegradable solid waste and spectral characteristics of the derived liquid digestate. Bioresour. Technol. 2014, 161, 69-77. [CrossRef] [PubMed]

40. Jimenez, J.; Gonidec, E.; Cacho Rivero, J.A.; Latrille, E.; Vedrenne, F.; Steyer, J.-P. Prediction of anaerobic biodegradability and bioaccessibility of municipal sludge by coupling sequential extractions with fluorescence spectroscopy: Towards ADM1 variables characterization. Water Res. 2014, 50, 359-372. [CrossRef] [PubMed]

41. Ganesh, R.; Torrijos, M.; Sousbie, P.; Steyer, J.P.; Lugardon, A.; Delgenes, J.P. Anaerobic co-digestion of solid waste: Effect of increasing organic loading rates and characterization of the solubilised organic matter. Bioresour. Technol. 2013, 130, 559-569. [CrossRef]

42. WTW GmbH. Supervision of BOD Measuring Systems According to DIN/ISO 9000 and GLP; WTW: Weilheim, Germany, 2010.

43. WTW GmbH. System OxiTop ${ }^{\circledR}$ Control: Operating Manual; WTW: Weilheim, Germany, 2004.

44. Hjorth, M.; Christensen, K.V.; Christensen, M.L.; Sommer, S.G. Solid-liquid separation of animal slurry in theory and practice. A review. Agron. Sustain. Dev. 2010, 30, 153-180. [CrossRef]

45. R Core Team. R: A Language and Environment for Statistical Computing; R Foundation for Statistical Computing: Vienna, Austria, 2014.

46. Husson, F.; Le, S.; Pages, J. Exploratory Multivariate Analysis by Example Using R. J. Stat. Softw. 2011, 40.

47. Ward, J.H. Hierarchical grouping to optimize an objective function. J. Am. Stat. Assoc. 1963, 58, 236-244. [CrossRef]

48. Oliveira, I.; Reed, J.P.; Abu-Orf, M.; Wilson, V.; Jones, D.; Esteves, S.R. The potential use of shear viscosity to monitor polymer conditioning of sewage sludge digestates. Water Res. 2016, 105, 320-330. [CrossRef]

49. Banks, C.J.; Chesshire, M.; Heaven, S.; Arnold, R. Anaerobic digestion of source-segregated domestic food waste: Performance assessment by mass and energy balance. Bioresour. Technol. 2011, 102, 612-620. [CrossRef] [PubMed]

50. Zirkler, D.; Peters, A.; Kaupenjohann, M. Elemental composition of biogas residues: Variability and alteration during anaerobic digestion. Biomass Bioenergy 2014, 67, 89-98. [CrossRef]

51. Sibil, R.; Berkun, M.; Bekiroglu, S. The comparison of different mathematical methods to determine the BOD parameters, a new developed method and impacts of these parameters variations on the design of WWTPs. Appl. Math. Model. 2014, 38, 641-658. [CrossRef]

52. Herrmann, C.; Idler, C.; Heiermann, M. Biogas crops grown in energy crop rotations: Linking chemical composition and methane production characteristics. Bioresour. Technol. 2016, 206, 23-35. [CrossRef] [PubMed]

53. Dandikas, V.; Heuwinkel, H.; Lichti, F.; Drewes, J.E.; Koch, K. Correlation between biogas yield and chemical composition of energy crops. Bioresour. Technol. 2014, 174, 316-320. [CrossRef] [PubMed]

54. Møller, H.B. Separation efficiency and particle size distribution in relation to manure type and storage conditions. Bioresour. Technol. 2002, 85, 189-196. [CrossRef]

55. Ganesh, R.; Torrijos, M.; Sousbie, P.; Lugardon, A.; Steyer, J.P.; Delgenes, J.P. Effect of increasing proportions of lignocellulosic cosubstrate on the single-phase and two-phase digestion of readily biodegradable substrate. Biomass Bioenergy 2015, 80, $243-251$. [CrossRef]

56. Yang, X.; Shang, C.; Lee, W.; Westerhoff, P.; Fan, C. Correlations between organic matter properties and DBP formation during chloramination. Water Res. 2008, 42, 2329-2339. [CrossRef] [PubMed]

57. Bieroza, M.Z.; Bridgeman, J.; Baker, A. Fluorescence spectroscopy as a tool for determination of organic matter removal efficiency at water treatment works. Drink. Water Eng. Sci. 2010, 3, 63-70. [CrossRef] 